\title{
Observation of neutral sodium above Mercury during the transit of November 8, 2006
}

\author{
A.E. Potter ${ }^{\mathrm{a}, *}$, R.M. Killen ${ }^{\mathrm{b}}$, Kevin P. Reardon ${ }^{\mathrm{c}, \mathrm{d}}$, T.A. Bida ${ }^{\mathrm{e}}$ \\ ${ }^{a}$ National Solar Observatory, Tucson, AZ 85719, United States \\ ${ }^{\mathrm{b}}$ NASA Goddard Space Flight Center, Greenbelt, MD 20771, United States \\ ${ }^{\mathrm{c}}$ INAF-Arcetri Astrophysical Observatory, Florence, Italy \\ ${ }^{\mathrm{d}}$ National Solar Observatory, Sunspot, NM 88349, United States \\ e Lowell Observatory, Flagstaff, AZ 86001, United States
}

\section{A R T I C L E I N F O}

\section{Article history:}

Received 1 December 2012

Revised 9 May 2013

Accepted 21 May 2013

Available online 6 June 2013

\section{Keywords:}

Mercury, atmosphere

Abundances, atmospheres

Atmospheres structure

\begin{abstract}
A B S T R A C T
We mapped the absorption of sunlight by sodium vapor in the exosphere of Mercury during the transit of Mercury on November 8, 2006, using the IBIS Interferometric BIdimensional Spectrometer at the Dunn Solar Telescope operated by the National Solar Observatory at Sunspot, New Mexico. The measurements were reduced to line-of-sight equivalent widths for absorption at the sodium $\mathrm{D}_{2}$ line around the shadow of Mercury. The sodium absorption fell off exponentially with altitude up to about $600 \mathrm{~km}$. However there were regions around north and south polar-regions where relatively uniform sodium absorptions extended above $1000 \mathrm{~km}$. We corrected the $0-600 \mathrm{~km}$ altitude profiles for seeing blur using the measured point spread function. Analysis of the corrected altitude distributions yielded surface densities, zenith column densities, temperatures and scale heights for sodium all around the planet. Sodium absorption on the dawn side equatorial terminator was less than on the dusk side, different from previous observations of the relative absorption levels. We also determined Earthward velocities for sodium atoms, and line widths for the absorptions. Earthward velocities resulting from radiation pressure on sodium averaged $0.8 \mathrm{~km} / \mathrm{s}$, smaller than a prediction of $1.5 \mathrm{~km} / \mathrm{s}$. Most line widths were in the range of $20 \mathrm{~mA}$ after correction for instrumental broadening, corresponding to temperatures in the range of $1000 \mathrm{~K}$.
\end{abstract}

(c) 2013 Elsevier Inc. All rights reserved.

\section{Introduction}

In 1985, sodium was discovered in emission in the exosphere of Mercury by resonance scattering of sunlight from the atoms (Potter and Morgan, 1985). It was realized that during a transit, the exospheric sodium should be detectable by absorption of sunlight. An attempt was made at the Hida Solar Observatory west of Osaka, Japan, to measure Mercury's sodium exosphere in absorption while Mercury transited the Sun's disk on 6 November, 1993. Observations were made at very high spectral resolution using a CCD detector, but no absorption was detected, probably due to seeing blur (Potter et al., 1994). The first successful observations of Mercury's sodium exosphere in absorption above the solar disk were made during the transit of Mercury May 7, 2003 using a 2-dimensional Fabry-Perot spectrograph with adaptive optics at the Vacuum Tower Telescope at Izana, Tenerife (Schleicher et al., 2004). The success of those observations, and availability of new instrumentation, prompted us to observe the Mercury sodium exosphere

\footnotetext{
* Corresponding author.

E-mail addresses: apotter@noao.edu (A.E. Potter), rosemary.killen@nasa.gov (R.M. Killen), kreardon@nso.edu (K.P. Reardon), tbida@lowell.edu (T.A. Bida).
}

during the transit of 8 November 2006. We used both the Main Spectrograph of the McMath-Pierce Solar Telescope at the Kitt Peak National Observatory, Arizona and the Interferometric BIdimensional Spectrometer (IBIS), a dual Fabry-Perot interferometer, at the Dunn Solar Telescope, Sunspot, New Mexico. IBIS is a tunable narrowband filter whose main components are two, air-spaced, $50 \mathrm{~mm}$ diameter Fabry-Perot interferometers (Cavallini, 2006; Cavallini and Reardon, 2006; Reardon and Cavallini, 2008; Righini et al., 2010). While spectra from the McMath-Pierce Main Spectrograph clearly showed absorption around the shadow of Mercury against the Sun, the data from the IBIS instrument were very superior, mostly due to the instrument's capability for short exposure times and high spectral resolution. Consequently, we report only the IBIS observations in this report.

Important scientific questions concerning the Mercury sodium exosphere can be addressed by observation of the transit. The surface density, zenith column density and temperature of exospheric sodium can be determined from measurements of the tangent absorption of sodium atoms above the Mercury surface during the transit. There is no other Earth-based method available to infer these fundamental properties of the sodium exosphere. Models of the sodium exosphere predict that the latitudinal variation of 
sodium along the terminator should differ between the dawn and dusk terminator (Leblanc and Johnson, 2010). The transit observation is the best way to test directly this prediction, since the entire terminator circumference is observed simultaneously. The temperature of the exosphere can be mapped in terms of line width, and used to test models for source processes. Solar radiation pressure accelerates sodium atoms in the antisunward direction, directly towards Earth during a transit. The predicted velocity achieved by the atoms is uncertain, since it depends on skip distances, energy of interaction with the surface, and so on. However, it can be measured directly during a transit, providing a real number against which to test theoretical models of the interaction of the sodium exosphere with solar radiation pressure and the surface.

\section{Data acquisition and processing}

\subsection{Data acquisition}

The IBIS instrument was operated during the transit to collect images of sodium $\mathrm{D}_{2}$ absorption around the Mercury silhouette during the period 19:26:27 UT to 20:18:33 UT on November 8, 2006 at the Dunn Solar Telescope at the National Solar Observatory at Sunspot, New Mexico. A total of 46 scans were obtained during this interval. The adaptive optics (AO) system (Rimmele, 2004) was utilized during all the observations, with the disk of Mercury itself serving as the reference target. The AO system and tip-tilt mirror kept Mercury fixed in the field of view, while the solar scene scrolled behind.

The observed spectral range from 5888.24 to $5891.13 \AA$ was sampled with a total of 159 spectral steps. In a $0.3 \AA$ A range covering the red wing of the line profile where the absorption feature from Mercury was expected, the line was incrementally scanned in wavelength steps of $0.0087 \AA$, while in most of the rest of the line, the sampling was in $0.030 \AA$ steps (the finer sampling was also used around the $5888.7 \AA$ telluric line). The sampling is illustrated in Fig. 1.

It took approximately $46 \mathrm{~s}$ to perform a single scan of the full line profile, during which time the disk of Mercury was moving at a rate of $0.085 \mathrm{arcsec} / \mathrm{s}$ relative to the surface of the Sun, shifting 4 arcsec against the background solar features. A total of 45 full

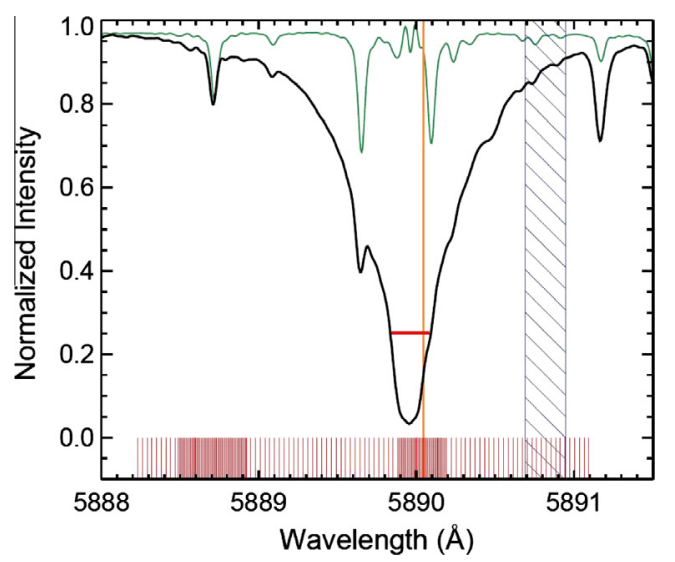

Fig. 1. This plot shows the spectral scan positions (red ticks along the bottom). The red bisector is the position where the line width was measured and corrected, the blue band is the interval that was used as the reference for the line depth normalization, and the orange line is the nominal position of the sodium absorption feature from the exosphere. The green spectrum at the top is the atmospheric absorption spectrum from the Kitt Peak atlas. (For interpretation of the references to color in this figure legend, the reader is referred to the web version of this article.) spectral scans were obtained. Each raw image was $1024 \times 512$ pixels, with a spatial scale of 0.083 arcsec per pixel.

\subsection{Data processing}

Residual distortions produced by the terrestrial atmosphere were removed by destretching each image with the subsequent image in the spectral scan to align the solar structures on $2.5 \times 2.5$ arcsec subfields (after removing the drift of the solar structures caused by the telescope tracking of Mercury). To improve the correction near the dark disk of Mercury, a radial gradient filter was calculated for each image, taking into account the fact that the observed planetary disk was not perfectly circular due to the distortions produced by the terrestrial atmosphere. The destretch vectors were calculated based on these radially filtered images and then applied directly to the original, unfiltered images. The spectral profile at each position in the field of view was then interpolated onto a common wavelength grid with 0.0087 A steps. As part of this interpolation, the radial instrumental blue shift was also removed. Because the disk of Mercury overlapped the optical center of the image, the blue shift was small near the limb $(\sim 1 \mathrm{~m} \AA)$ of Mercury. The images were then trimmed spatially to produce an $x, y, \lambda$ data cube with dimensions of [800,512,329] pixels.

We selected the 26 spectral scans that showed the best seeing conditions in the wavelengths expected for the exosphere absorption signature. These spectral data cubes were summed to produce a single spectral scan with the magnitude of the intensity fluctuations due to solar structures greatly reduced. However there were still systematic variations in the depth, width, and intensity of the solar sodium $\mathrm{D}_{2}$ line at different points in the field due to the nature of the solar structures sampled at each position. In order to isolate the absorption feature produced by the Hermean exosphere, it was necessary to remove these variations. This is especially critical because during the transit the absorption feature from the exosphere is shifted approximately $0.1 \AA$ to the red of the solar line core due to Mercury's motion relative to the Sun. This shift places the feature at approximately the wavelength where the intensity crosstalk produced by inherent Doppler-shifts or line width variations in the solar line profile will be greatest.

For all points in the field of view the total line depth (between the line core minimum intensity and the mean intensity in the red wing of the profile in the range +0.75 to $1.0 \AA$ ), the line core position, and the line width at $25 \%$ of the continuum intensity (approximately $\pm 0.12 \AA$ from the line core) were measured. This intensity level was chosen for the bisector measurement in order to minimize disturbances from telluric lines present in both the red and blue wings of the line profile. The normalization to the nearby red wing intensity also provided a correction for the intensity changes at points near the limb of Mercury as points were covered or uncovered during the $20 \mathrm{~s}$ it took to scan in wavelength between the core and red wing.

A reference profile was generated by averaging the line profiles in an annulus between 4 and $16 \operatorname{arcsec}(1960$ and $7820 \mathrm{~km}$ ) above the limb of Mercury. Prior to averaging, each individual profile was shifted to align the line position (measured in both the wing and core) with the average position, the total line depth normalized to unity, and scaled to have line width equal to the average across all the profiles. This gave an average profile that was not broadened by line shifts or width variations.

Then for the spectrum observed at each point in the field of view, this average profile was shifted in wavelength, and scaled in intensity and line width to match each observed spectrum. The observed profile was then divided by the scaled reference profile to remove the solar absorption and derive the profile of the relative absorption, if present. 
An example of the comparison of the reference solar signal with signal in the absorption region as a function of wavelength for one position in the field of view is shown in Fig. 2.

The Hermean absorption feature as illustrated in Fig. 2 was analyzed to compute equivalent width, Earthward velocity, and line width for each location in the instrument field of view. Earthward velocities for sodium atoms were calculated from the difference between the centroid wavelength of the absorption line and its predicted position derived from the Mercury orbital ephemeris (5890.06 A). The wavelength calibration needed for these calculations was derived from positions of atomic emission lines from a standard lamp. The line width was determined by least squares fitting of a Gaussian distribution, $\left(\exp -\left(\lambda-\lambda_{o}\right)^{2} / 2 \sigma^{2}\right)$, where $\lambda_{o}$ is the centroid wavelength, and $\sigma$ is the standard deviation, defined here as width for the absorption line) to the spectral profile of the absorption line. The data from the spectral-spatial images were combined to yield equivalent width, velocity, and line width images of the entire planet.

In order to test the validity of our approach to separating the solar and Hermean absorption features, we also applied an independent analysis based on Proper Orthogonal Decomposition (Vecchio et al., 2005a, 2005b). The normalized, averaged spectra, as described above, for the full field of view were broken down into separate components that were only determined by the algorithm. This approach separated the background solar spectra from Mercury's absorption component, which was found only around the limb of the planet. The distribution and strength and noise characteristics of the resulting absorption profiles were similar to our previous results, confirming the reliability of our processing method.

The image cube can be sliced into images having two spatial dimensions $(800 \times 512)$, each one a monochromatic image of Mercury silhouetted against the Sun. There was one such image for each of the wavelengths (329 total) included in the spectral range of the scans. An example of one of these images is shown in Fig. 3.

The image in Fig. 3 was chosen for a wavelength near the center of the Hermean sodium absorption, so as to show dark regions surrounding the disk of Mercury which represent absorption by sodium in the Mercury exosphere. The mottled appearance of the image away from the absorption region is residual noise produced by uncorrected Doppler shifts and broadening of solar spectral lines.

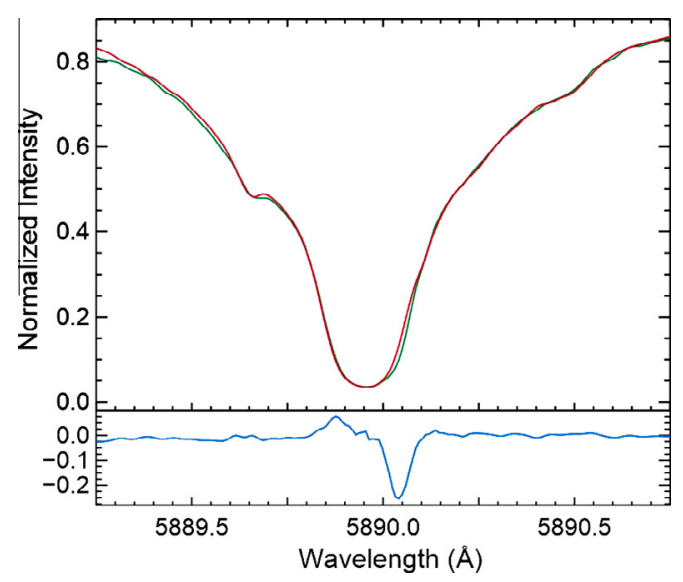

Fig. 2. Here is shown spectral profiles for regions with and without sodium absorption. The green line is the sodium Fraunhofer line spectrum taken from a point above the north pole of Mercury. The red line is the reference spectrum taken away from Mercury. The blue line in the bottom half of the figure is the spectral ratio used to calculate the equivalent width, line width and Doppler shift for sodium absorption. (For interpretation of the references to color in this figure legend, the reader is referred to the web version of this article.)

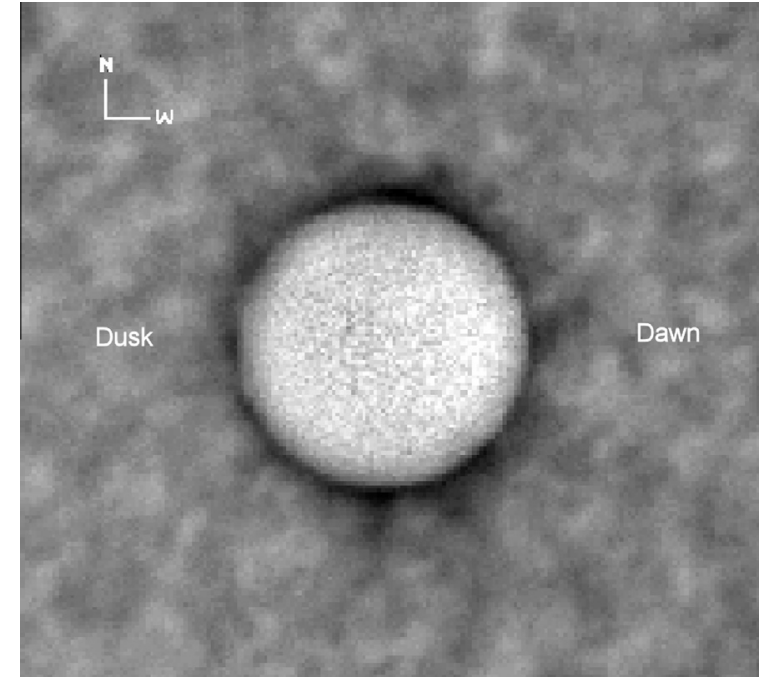

Fig. 3. Image of Mercury silhouetted against the Sun at maximum sodium absorption wavelength. This is an average image from 19 scans, and the reference spectrum from the Sun has been divided out. The wavelength for this image was chosen to be near the center of sodium absorption in the exosphere of Mercury, so that the dark regions near the perimeter of Mercury represent the effect of sodium absorption. This disk of Mercury appears bright in this image (although in reality it is dark) as an artifact of the division by the reference solar spectrum at those ondisk points.

\section{Data analysis and discussion}

\subsection{Equivalent widths around the planet}

The equivalent width in units of $\mathrm{mA}$ was calculated at each point in the field of view as described in the previous section. Results were used to produce an image of the equivalent width distribution around the planet, as is shown in Fig. 4.

The image is oriented as the planet is seen in the sky (north up, west to the right). West is the dawn terminator, east is the dusk terminator. Mercury was moving left to right across the Sun at the time of this observation. Equivalent widths near the planet surface ranged between $2 \mathrm{~mA}$ and $18 \mathrm{~mA}$. This image includes regions far from the planet where there is no sodium absorption, and the calculated equivalent width values provide an estimate of the noise resulting from variations of solar intensity. The rms value of this noise was $1.6 \mathrm{~mA}$.

As seen in the figure, the sodium absorption is greatest over the north and south poles, with a somewhat wider extent of high absorption over the south polar regions. Absorption over the dawn (west) and dusk (east) terminator are only slightly different, with the dusk terminator signal being a little larger. The irregularities seen in the distribution of sodium around the planet are believed to be real effects.

\subsubsection{Altitude variation of equivalent width}

In order to quantify the distribution of sodium absorption around the planet, we analyzed the radial distribution of sodium absorption above the surface. The image of Fig. 4 was sampled in $15^{\circ}$ arcs starting at an arc centered at $0^{\circ}(\mathrm{N})$, and proceeding in $15^{\circ}$ arcs around the image. Each arc was divided into radial steps, each increasing by one pixel. Data points in each radial step were averaged and plotted into a polar coordinate system to yield an image in which the sodium absorption values were radial. In the course of this averaging, the standard deviation from the mean value was calculated for each arc. Values ranged from 0.2 to $0.8 \mathrm{~mA}$, in reasonable agreement with expectations from the rms noise value in the region outside the Mercury image, considering that each 


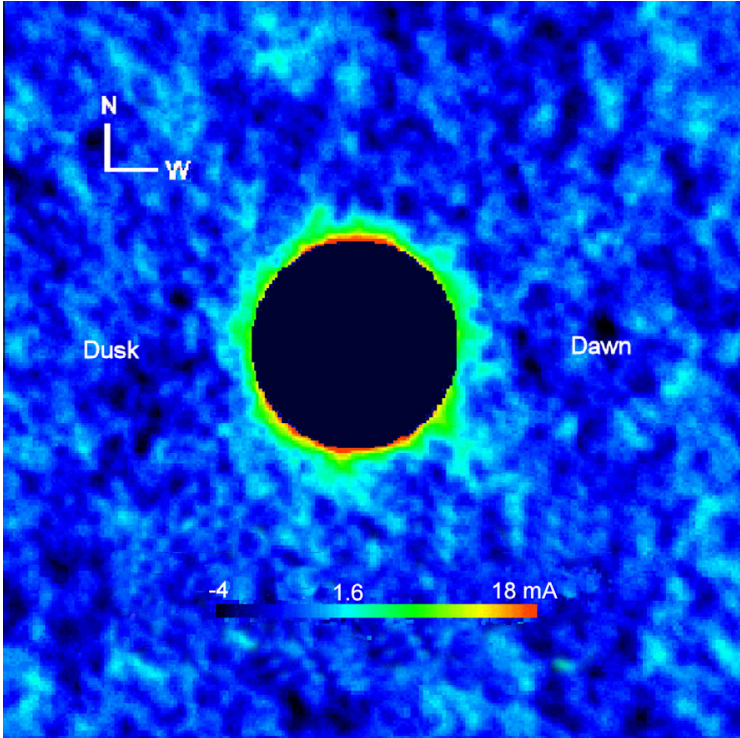

Fig. 4. Map of the distribution of equivalent width for sodium absorption around the disk of Mercury. The map shows the noise spectrum outside the region of sodium absorption that is the result of fluctuations in solar intensity resulting from turbulence. The rms noise level in this region was $1.6 \mathrm{~mA}$, and this value was chosen as the threshold for appearance of significant absorption. The general appearance of the map is similar to that found by Schleicher et al. (2004), in that the strongest absorptions are seen around the north and south pole. However, there is a difference in that the absorptions on the dawn (west) terminator are not much different than on the dusk (east) terminator. Schleicher et al. (2004) found the absorption over the dusk terminator to be much smaller than over the dawn terminator.

arc near the surface averaged 17-18 points, increasing to 28 points at $2000 \mathrm{~km}$. Data points for which the average equivalent width was less than the rms deviation of the noise $(1.6 \mathrm{~mA})$ were discarded, and values were calculated up to an altitude of $2000 \mathrm{~km}$. The image in Fig. 5 shows the result, in which sodium absorption has been averaged in $15^{\circ}$ segments around the planet.

We found that the radial distribution of sodium absorption could be represented reasonably well by an exponential up to altitudes of about $600 \mathrm{~km}$. An example for the altitude distribution of sodium absorption is shown in Fig. 6 which is a logarithmic plot of equivalent width and rms errors per altitude step versus altitude for the $15^{\circ}$ segment over the North Pole. The altitude step for these data was $39.35 \mathrm{~km}$. At intermediate altitudes, the equivalent width deviates slightly in a positive direction from the linear fit, and at the highest altitudes it falls off considerably in a negative direction from the linear fit. This was a pattern seen for most of the altitude profiles.

However, for several arcs in the solar polar region (centered at $150^{\circ}, 165^{\circ}, 180^{\circ}$ and $225^{\circ}$ ) and for two arcs in the north polar region (centered at $15^{\circ}$ and $45^{\circ}$ ), sodium absorption continues beyond $600 \mathrm{~km}$, on up to altitudes of $1000 \mathrm{~km}$ or higher. The highaltitude sodium displays a cloud-like structure, as shown in Fig. 7, which is an enlarged cutout of a region in the $225^{\circ}$ sector taken from the un-averaged image in Fig. 4. The appearance of weak sodium patches at altitudes above the expected range of the exosphere is an unexpected phenomenon. The "spatial wavelength" (peak-to-peak distance) of these patches approximately matches the "wavelength" of the noise well away from Mercury, suggesting that this effect is simply a manifestation of stronger patches of noise close to the limb. However, for some regions at high north and south latitudes, the high altitude absorption survives averaging over $15^{\circ}$ arcs, which suggests that they may be real phenomena.

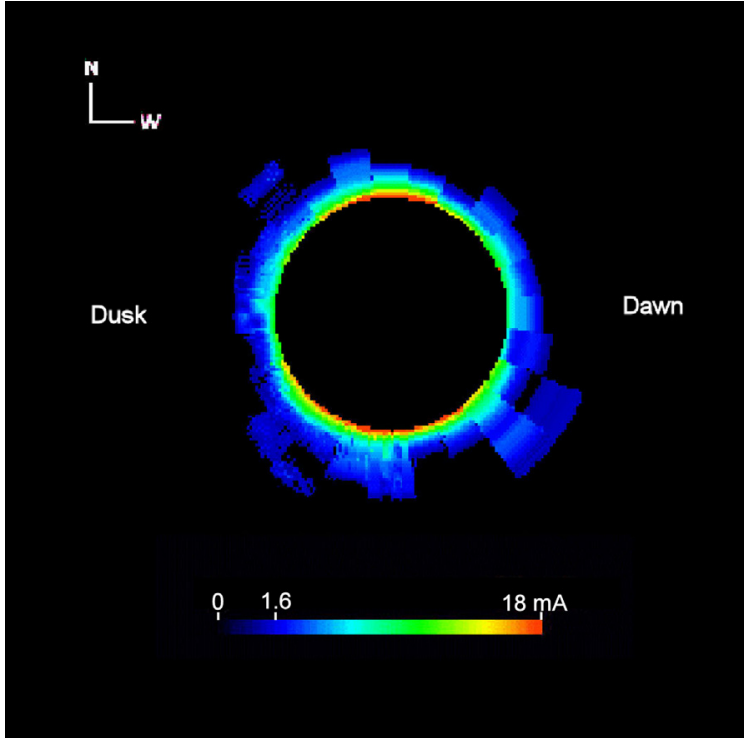

Fig. 5. Image derived from the map shown in Fig. 4. It shows the radial distribution of equivalent widths that have been averaged over $15^{\circ}$ increments around the disk of Mercury. Only equivalent widths greater than $1.6 \mathrm{~mA}$, the rms noise level, are shown.

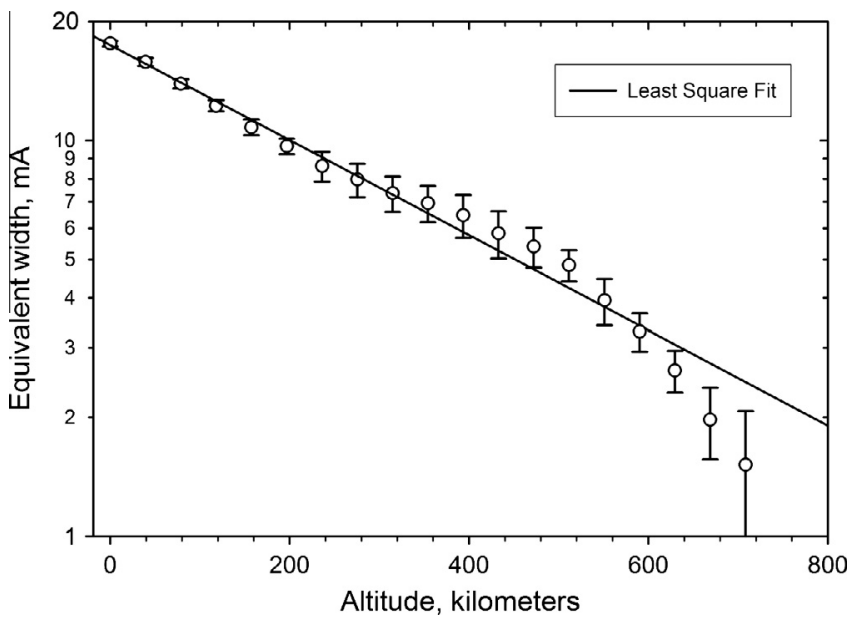

Fig. 6. This plot is a sample of numerical data from the radial distribution map of Fig. 5. The equivalent width for sodium absorption from a $15^{\circ}$ segment above the north pole is plotted against altitude above the surface. In this logarithmic plot, the $\log$ of the equivalent width falls off linearly with increasing altitude, thus the equivalent width falls off exponentially with increasing altitude. The sodium absorption measured closest to the Mercury shadow was assumed to be at the surface. The equivalent width at the surface and the slope of the altitude dependence (the apparent scale height) were calculated for each of the $15^{\circ}$ segments around the circumference of the planet.

The altitude dependence of equivalent widths from the $225^{\circ}$ arc in the averaged image of Fig. 5 is plotted in Fig. 8 up to an altitude of $1500 \mathrm{~km}$.

Sodium absorption stops decreasing above $800 \mathrm{~km}$, then rises to a maximum near $1200 \mathrm{~km}$ before decreasing again. The high-altitude sodium above $600 \mathrm{~km}$ may be from a different source than the low-altitude sodium. It may represent the small population of high energy sodium in the sodium tail of Mercury, which Potter and Killen (2008) showed were generated with energies $\sim 3 \mathrm{eV}$. Data from the MESSENGER mission have shown that northern and southern polar-regions are open to direct particle impact by the solar wind (Anderson et al., 2011; Winslow et al., 2012). The 


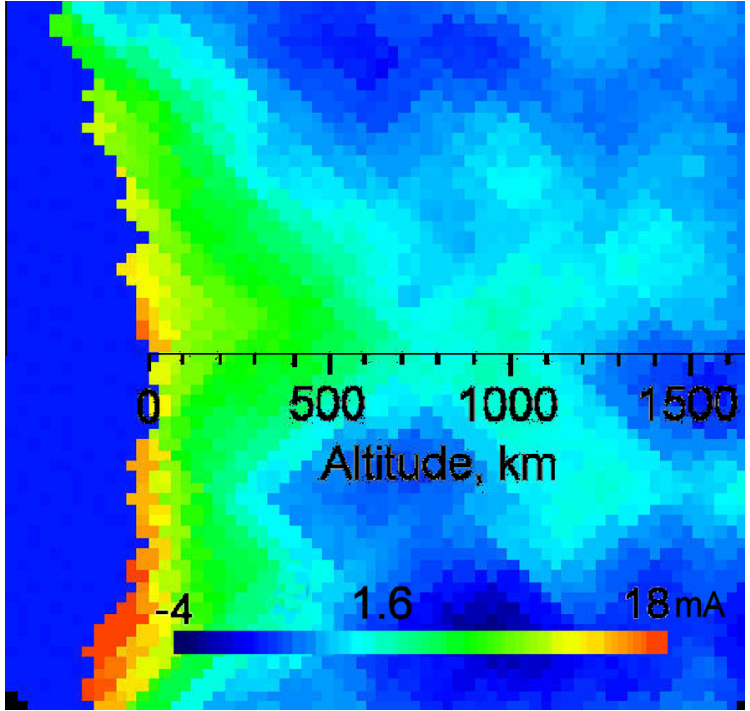

Fig. 7. A close-up view of one of the high altitude sodium clouds seen in Fig. 4 near the position angle of $225^{\circ}$. The threshold for useful signal of $1.6 \mathrm{~mA}$ is marked on the color bar.

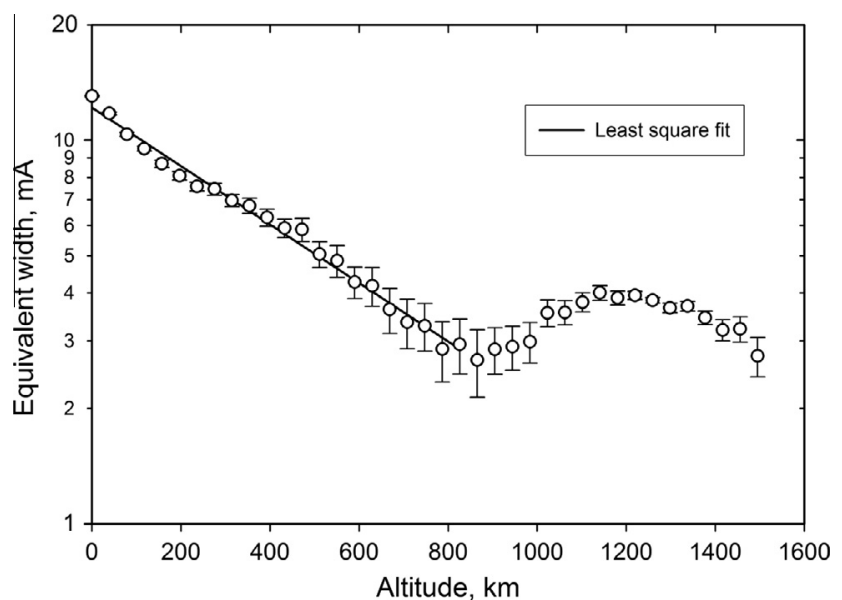

Fig. 8. This is a plot of equivalent width for the sector at $225^{\circ}$, which includes one of the sodium clouds, extended up to $1500 \mathrm{~km}$. The log plot is linear up to about $800 \mathrm{~km}$. Sodium absorption continues to higher altitudes, increasing to a maximum at about $1200 \mathrm{~km}$ before falling off.

plasma precipitating through the southern cusp may exceed that in the north by a factor of four as a consequence of the planet's offset magnetosphere (Winslow et al., 2012).

Returning to the sodium absorptions seen from the surface up to $600 \mathrm{~km}$, their altitude distributions can be represented with a simple exponential, as follows:

$I=I_{0} \exp -\left(\frac{h}{h_{0}}\right)$

where $I$ is the observed equivalent width, $I_{0}$ is the observed equivalent width per altitude step at the surface (zero altitude), $h$ is the altitude, and $h_{0}$ the apparent scale height.

We fitted Eq. (1) to the $15^{\circ}$ arc observations (including the errors at each radial step) around the planet to yield the tangent surface equivalent widths $I_{0}$ and apparent scale heights $h_{0}$, along with error estimates, as a function of position angle (defined as the angle eastward from north). Results are listed in Table 1.
Equivalent widths are plotted in Fig. 9 as a polar plot of surface equivalent widths versus position on the planet.

Maximum values are at the poles. At the equator, the surface absorption was about $30 \%$ less at the dawn terminator than at the dusk terminator. The surface absorption averaged over a $60^{\circ}$ range centered at the equator is about $10 \%$ less over the dawn terminator than over the dusk terminator. The difference should not be overemphasized considering the uncertainties of the measurement.

Fig. 10 is a polar plot of the apparent scale height around the circumference of the planet.

The apparent scale heights over polar regions are similar, with the smallest values offset dawn-ward (W) $15^{\circ}$. Scale heights over the dawn hemisphere average about $100 \mathrm{~km}$ larger than over the dusk hemisphere.

\subsubsection{Effect of atmospheric seeing on the equivalent widths and scale heights}

Terrestrial atmospheric seeing significantly degrades the altitude profile, causing the observed equivalent widths to be less than the true values, and scale heights to be larger than the true values. It is possible to make approximate corrections for seeing effects provided the profile of the seeing blur is known. We determined the seeing function for this observation by analysis of the profile of the Mercury shadow at wavelengths away from the absorption region. The resulting point spread profile is shown in Fig. 11 as black line plot. The profile was approximated as the sum of two Gaussians with widths of 0.41 arcsec and 2.3 arcsec, and the first having three times the magnitude of the second (illustrated as a red line plot in Fig. 11).

In principle, we can recover the original altitude profiles by deconvolving the observed profiles with the seeing profile. However, this proved to be impractical, as noise is greatly magnified in the process of deconvolution. We chose to estimate the true altitude profiles, assumed to be exponential, by convolving model altitude profiles with the seeing profile, and varying the model altitude profile until the result from the convolution matched the observed profile. The convolution routine was taken from Press et al. (1992). The seeing profile used for the convolution spanned 4 arcsec, and was normalized to a total of unity before the convolution. An example is illustrated in Fig. 12, where the observed altitude profile is compared with the model profile before and after convolution with seeing.

The results from this analysis are listed in Table 2 where corrected equivalent widths and scale heights are listed. We assumed that the error values scaled linearly with the equivalent widths and scale heights.

A polar plot of the corrected tangent equivalent widths is shown in Fig. 13.

The corrected surface equivalent widths are in the range 3-15 times larger than the measured surface values, depending on the apparent scale height. The correction for seeing is not a linear process, so that sites with relatively small scale heights display a large correction, while sites with large scale heights display a smaller correction. The result is that the polar pattern for corrected equivalent widths looks somewhat different than the uncorrected pattern. The difference between surface equivalent widths at the dawn and dusk terminators is accentuated relative to the uncorrected values, with dawn-side values about half the dusk-side values. This interesting difference suggests that there is a basic difference between sodium production at the dawn and dusk terminators. There are prominent peaks $30^{\circ}$ dawn-ward at both north and south poles, suggesting the production of sodium at high latitudes, in general agreement with ground-based observations (Leblanc et al., 2009a, 2009b; Mangano et al., 2009). 
Table 1

Apparent equivalent widths at the surface and corresponding scale heights.

\begin{tabular}{|c|c|c|c|c|}
\hline Position angle $\left({ }^{\circ}\right)$ & Apparent equivalent width (mA) & Error, apparent equivalent width & Apparent scale height (km) & Error, apparent scale height \\
\hline $0 N$ & 17.4 & 0.2 & 361.2 & 8.3 \\
\hline 15 & 15.8 & 0.2 & 298.0 & 2.9 \\
\hline 30 & 13.8 & 0.2 & 242.7 & 3.1 \\
\hline 45 & 10.3 & 0.2 & 387.4 & 39.7 \\
\hline 60 & 10.4 & 0.1 & 549.5 & 9.8 \\
\hline 75 & 8.5 & 0.1 & 491.1 & 8.6 \\
\hline $90 \mathrm{E}$ & 9.9 & 0.1 & 834.3 & 65.7 \\
\hline 105 & 10.2 & 0.1 & 555.4 & 91.3 \\
\hline 120 & 13.8 & 0.1 & 424.6 & 12.6 \\
\hline 135 & 15.2 & 0.2 & 526.4 & 21.9 \\
\hline 150 & 15.7 & 0.1 & 337.2 & 5.2 \\
\hline 165 & 16.3 & 0.1 & 267.0 & 3.4 \\
\hline $180 \mathrm{~S}$ & 16.3 & 0.2 & 460.4 & 22.3 \\
\hline 195 & 15.2 & 0.1 & 396.9 & 14.7 \\
\hline 210 & 15.6 & 0.1 & 355.6 & 9.8 \\
\hline 225 & 12.6 & 0.1 & 401.6 & 16.2 \\
\hline 240 & 11.48 & 0.1 & 315.2 & 4.2 \\
\hline 255 & 5.8 & 0.3 & 287.7 & 5.6 \\
\hline $270 W$ & 7.3 & 0.1 & 487.1 & 18.7 \\
\hline 285 & 8.7 & 0.1 & 564.6 & 41.0 \\
\hline 300 & 9.5 & 0.1 & 446.2 & 16.6 \\
\hline 315 & 10.4 & 0.3 & 383.0 & 17.6 \\
\hline 330 & 12.5 & 0.1 & 354.2 & 17.6 \\
\hline 345 & 17.9 & 0.1 & 346.5 & 18.8 \\
\hline
\end{tabular}

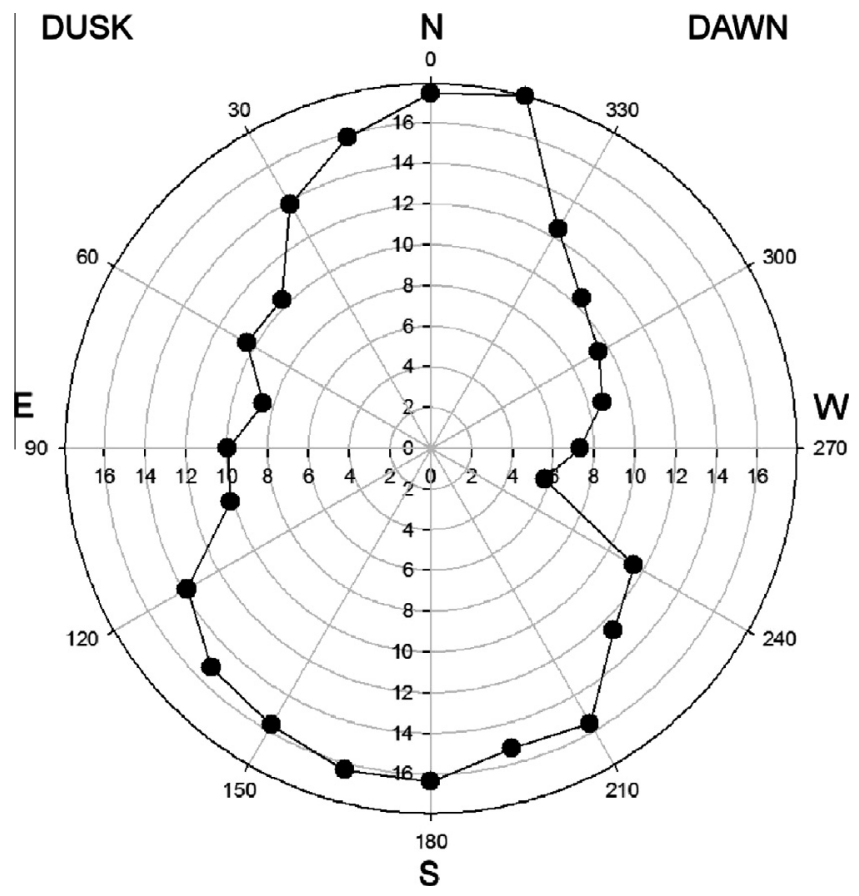

Fig. 9. Polar plot of equivalent width at the surface as a function of position angle around the planet. Maximum absorptions are seen over the polar regions. The dawn side equivalent width in equatorial regions is somewhat smaller than on the dusk side. The position angles are measured in the eastward direction from the north pole. The amplitudes of the surface equivalent widths mirror the intensity variations seen in Figs. 4 and 5. Values in this plot were taken from Table 1.

A polar plot of the corrected scale height values is shown in Fig. 14.

The difference between dawn and dusk scale heights increased from the uncorrected case, in a direction opposite from the surface equivalent widths. The average scale height on the dawn-side was about twice the average of the dusk-side values.

The total equivalent width can be found by integrating the altitude distribution of equivalent width. The result of this calculation

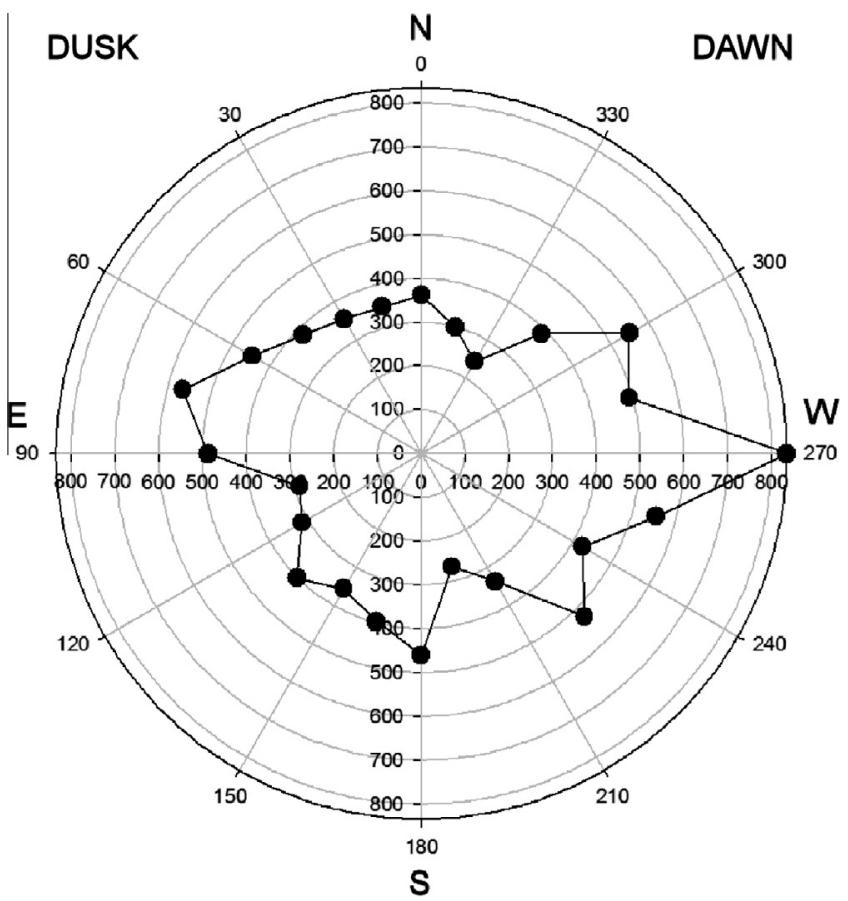

Fig. 10. Polar plot of the apparent scale height for sodium absorption around Mercury as a function of position angle. Dawn-side (W) heights are larger than dusk-side (E) heights by about a hundred kilometers. Values in this plot were taken from Table 1.

is shown in Table 2 and in Fig. 15, where total equivalent width is plotted as a function of position angle.

The total equivalent width (summed over altitude) is largest over the poles, and is smaller and approximately equal between the dawn and dusk equatorial terminators. Since the total sodium is not much different between dawn and dusk equatorial terminators, we suggest that the difference between surface equivalent widths between dawn and dusk terminators is the result of generation of sodium with higher temperatures on the dawn side, resulting in higher scale heights, as observed. 


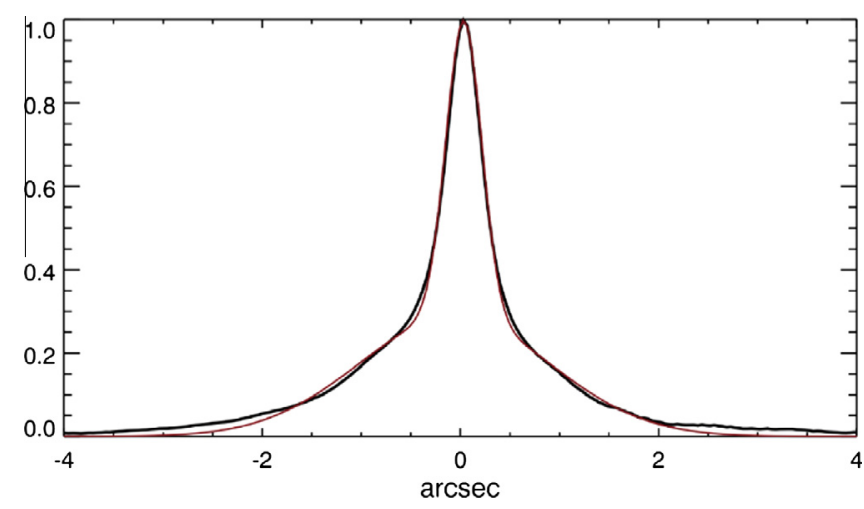

Fig. 11. The point spread function for atmospheric seeing during this observation. The profile was derived from analysis of the edge of the Mercury shadow at a wavelength where no sodium absorption was present. The black line represents the measured point spread function, and the red line represents the sum of two Gaussians, one with a width of 0.41 arcsec and the other 2.3 arcsec, the first having three times the magnitude of the second. (For interpretation of the references to color in this figure legend, the reader is referred to the web version of this article.)

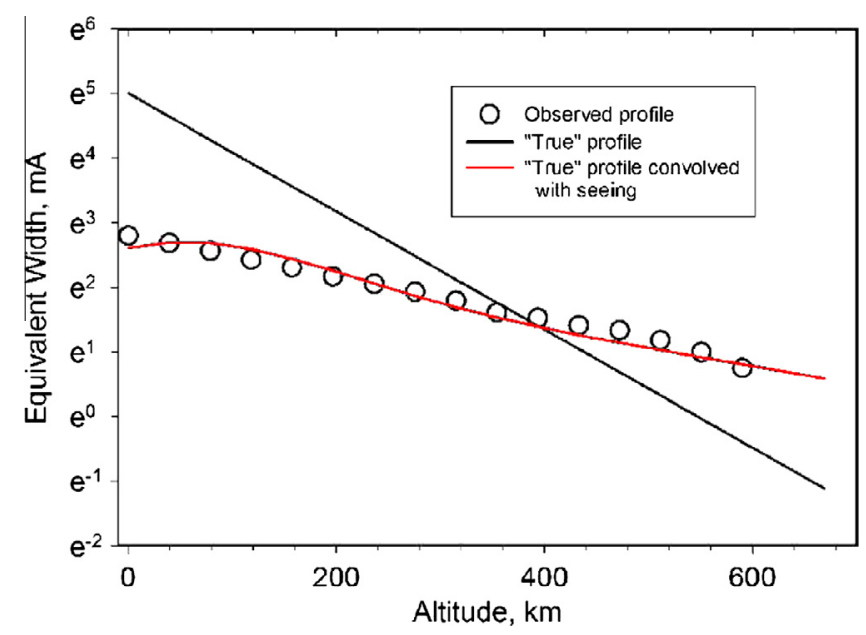

Fig. 12. This plot is an example of the application of the point spread function. It shows lines for a trial altitude profile before convolution (black line) and after convolution with the seeing function (red line), plotted over data points for an observed altitude profile over the north polar region. The decay of the convolved line into the shadow of Mercury is not plotted. (For interpretation of the references to color in this figure legend, the reader is referred to the web version of this article.)

The high-latitude enhancements illustrated in Figs. 13 and 15 could be associated with either sputtering, ion-enhanced photonstimulated desorption (ion-enhanced PSD) (Sarantos et al., 2010), or electron-stimulated desorption associated with the locus of open field lines near the poles. We therefore obtained estimates of the interplanetary magnetic field (IMF) and solar wind ram pressure at Mercury for the month bracketing the observation from the Community Coordinated Modeling Center (CCMC) at NASA Goddard Space Flight Center. The ENLIL model was run by the CCMC, giving modeled IMF values of $[\mathrm{Br}, \mathrm{B} \phi, \mathrm{Bz}]=[-14,12,-0.03]$. Both the solar wind ram pressure $(\sim 22 \mathrm{nPa})$ and the total magnetic field strength (14 nT) were high on 8 November, 2006, indicating an open magnetosphere. The negative Bz would be consistent with a larger cusp in the southern hemisphere as observed by MESSENGER (Winslow et al., 2012). We have evidence for higher densities near the poles but not for higher temperatures at the same places where we see enhanced surface densities. Although the southern cusp is probably larger than the northern cusp at this time, the northern smooth plains are regions of enhanced $\mathrm{Na}$ abundance in the rock, and probably cover a region a factor of two larger than the smooth plains in the south (Evans et al., 2013). Radiation pressure accelerates Na atoms antisunward, away from the Sun, but in this case because Mercury's heliocentric radial velocity is toward the Sun, and radiation pressure decreases as the antisunward velocity of the Na atom increases (Killen et al., 2009). Without a measurement of the Na tail, that region antisunward of the planet where the $\mathrm{Na}$ atoms have been accelerated by radiation pressure to escape velocity (e,g Schmidt et al., 2010, 2012; Potter and Killen, 2008; Baumgardner et al., 2008), we cannot conclusively determine the energy of the source atoms. However, since an ion sputter source would be hotter than we observe, it is possible that the enhancements are due to electron sputtering or ion-enhanced photon stimulated desorption (PSD), which have a source temperature consistent with that observed. A process in which solar wind protons liberate the sodium from the rock and those Na atoms are later released by PSD, called ion-enhanced PSD, was first suggested by Potter et al. (2000) to explain the behavior of the lunar exosphere during passage through the Earth's magnetotail. It was suggested to be the process maintaining the Hermean Na exosphere by Mura et al. (2009) in their analysis of the observations of the transit of 2003. Electrons have been observed streaming onto the planet at both high northern latitudes and at the equator (Ho et al., 2012).

\subsubsection{Zenith column densities derived from line-of-sight column densities}

Equivalent width values for the 2006 transit can be converted to line-of-sight column densities of sodium atoms by multiplying them by the Doppler-shifted solar flux of $7.8167 \times 10^{12}$ photons/ $\mathrm{cm}^{2} / \mathrm{s} / \mathrm{A}$ (Chance and Kurucz, 2010) and dividing by the $g$ value of 14.9 photons/atoms/s appropriate for the Doppler shifted wavelength of the sodium absorption (Killen et al., 2009), (taking into account antisunward velocity imparted by solar radiation pressure) to yield a conversion factor of $5.25 \times 10^{8}$ atoms $/ \mathrm{cm}^{2} \mathrm{~mA}$. The corrected equivalent widths (units of $m \AA$ ) derived from the absorption profiles can be expressed as tangent or line-of-sight (LOS) column densities by multiplying by this factor. The LOS column density is the summation of absorptions over the entire altitude range of the exosphere, as it represents the view along a straight line tangent to the planetary sphere. However it is possible to derive a complete characterization of the exosphere-the zenith column density, surface density, and temperature-from the altitude distribution of LOS column density using the analysis given by Chamberlain and Hunten (1987), as follows:

The density, $n(r)$, is the product of the barometric density and a partition function, $\zeta$, describing how the particles are partitioned among the various classes of orbits: ballistic, satellite and escaping. The integrated density along a line of sight at direction $\theta\left(r_{1}\right)$ along a chord that has its closest approach to the planet at $r_{1}$ from the planet center is

$N\left(\mu_{1}, \lambda_{1}\right)=\int_{r_{1}}^{\infty} n(r) \sec (\theta) d r$

where $\mu=\cos (\theta)$, and the number density $n$ at a radial distance from the planetary center, $r$, is

$n(r)=n_{c} e^{\left(\lambda_{e}-\lambda\right)} \varsigma(\lambda)$

where the subscript $c$ refers to the "critical level", as defined by Chamberlain and Hunten (1987) in their formulation of this problem. For the case at hand, it refers to the Mercury surface. The symbol $\lambda$ refers to the gravitational potential energy.With a change of variables, the integral can be taken over the gravitational potential $\lambda$ 
Table 2

Corrected equivalent widths at the surface and corresponding scale heights

\begin{tabular}{|c|c|c|c|c|c|c|}
\hline $\begin{array}{l}\text { Position angle } \\
\left({ }^{\circ}\right)\end{array}$ & $\begin{array}{l}\text { Equivalent width } \\
(\mathrm{mA})\end{array}$ & $\begin{array}{l}\text { Error, equivalent } \\
\text { width }\end{array}$ & $\begin{array}{l}\text { Scale height } \\
(\mathrm{km})\end{array}$ & $\begin{array}{l}\text { Error, scale } \\
\text { height }\end{array}$ & $\begin{array}{l}\text { Total equivalent width } \\
(\mathrm{mA})\end{array}$ & $\begin{array}{l}\text { Error, total equivalent } \\
\text { width }\end{array}$ \\
\hline $0 \mathrm{~N}$ & 119.3 & 2.9 & 156.6 & 6.3 & 18688.0 & 278.6 \\
\hline 15 & 113.5 & 6.7 & 145.3 & 14.4 & 16496.3 & 561.5 \\
\hline 30 & 96.7 & 3.7 & 151.3 & 9.5 & 14628.6 & 330.3 \\
\hline 45 & 66.5 & 2.6 & 172.9 & 12.7 & 11492.7 & 365. \\
\hline 60 & 59.7 & 1.2 & 217.0 & 10.8 & 12947.0 & 361.4 \\
\hline 75 & 43.6 & 1.5 & 286.8 & 20.2 & 12506.3 & 411.8 \\
\hline $90 \mathrm{E}$ & 54.4 & 0.9 & 243.0 & 11.2 & 13220.5 & 370.1 \\
\hline 105 & 98.7 & 3.7 & 97.4 & 4.7 & 9808.7 & 83.9 \\
\hline 120 & 113.3 & 2.3 & 120.3 & 3.4 & 13636.1 & 97.1 \\
\hline 135 & 93.9 & 3.2 & 186.4 & 12.8 & 17510.8 & 572.9 \\
\hline 150 & 109.0 & 3.3 & 152.4 & 7.5 & 16605.7 & 294.8 \\
\hline 165 & 101.8 & 2.9 & 183.1 & 10.4 & 18644.3 & 492.0 \\
\hline $180 \mathrm{~S}$ & 92.0 & 2.2 & 226.3 & 14.0 & 20813.2 & 751.8 \\
\hline 195 & 175.8 & 5.5 & 79.5 & 3.0 & 13978.6 & 62.0 \\
\hline 210 & 115.9 & 2.3 & 138.0 & 4.1 & 16001.9 & 145.8 \\
\hline 225 & 66.7 & 1.2 & 266.0 & 12.0 & 17755.4 & 478.3 \\
\hline 240 & 68.0 & 1.3 & 202.5 & 8.5 & 13761.1 & 302.5 \\
\hline 255 & 29.8 & 2.5 & 281.9 & 43.3 & 8403.6 & 480.6 \\
\hline $270 W$ & 23.2 & 4.4 & 382.7 & 10.1 & 8890.3 & 1509.8 \\
\hline 285 & 47.3 & 0.4 & 245.4 & 5.2 & 11603.2 & 149.6 \\
\hline 300 & 49.1 & 0.4 & 278.8 & 5.2 & 13694.0 & 145.3 \\
\hline 315 & 66.5 & 5.0 & 176.2 & 28.1 & 11718.9 & 851.7 \\
\hline 330 & 192.5 & 8.5 & 57.9 & 2.8 & 11146.8 & 25.0 \\
\hline 345 & 162.2 & 2.8 & 106.1 & 2.4 & 17208.5 & 79.6 \\
\hline
\end{tabular}

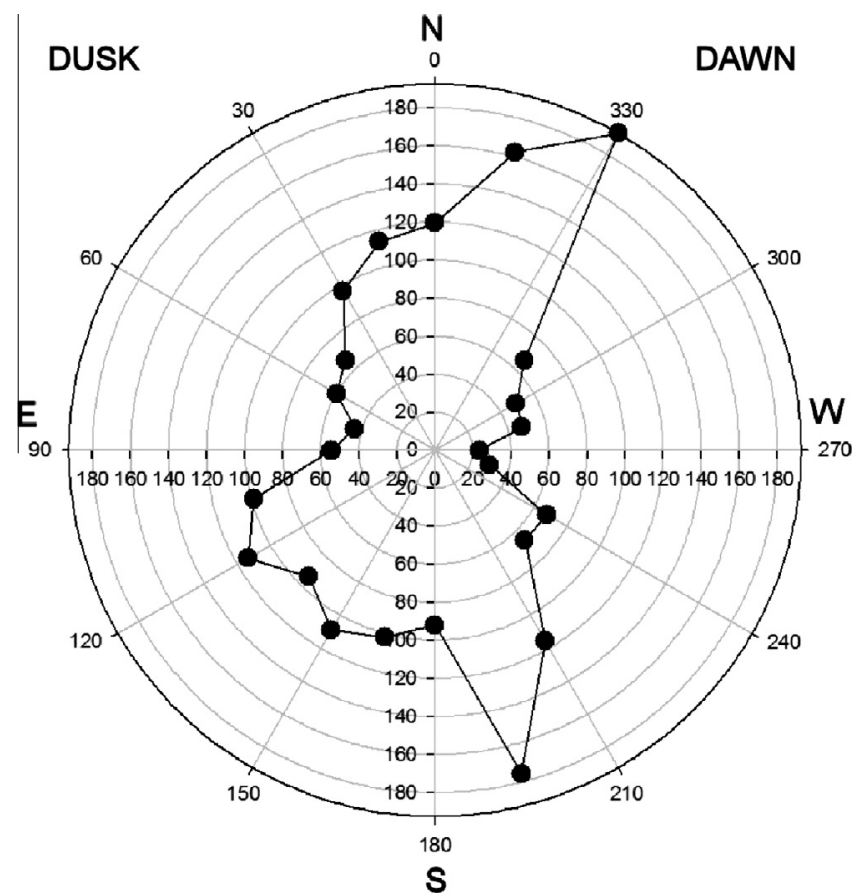

Fig. 13. This polar plot shows the corrected line-of-sight equivalent widths at the surface as a function of position angle around the disk of Mercury. Equivalent widths on the dawn side are about half the values seen on the dusk side. Peaks in north and south polar regions are evident, tilted towards the dawn hemisphere. Values in this plot were taken from Table 2.

$N\left(\mu_{1}, \lambda_{1}\right)=n_{c} e^{-\lambda_{c}} \lambda_{1} r_{1} \int_{0}^{\lambda_{1}} \frac{e^{\lambda} \varsigma(\lambda)}{\lambda^{2} \mu\left(\lambda, \lambda_{1}\right)} d \lambda$

and expressed in terms of the function, $K$

$N\left(r_{1}, \lambda_{1}\right)=n\left(r_{1}\right) H_{1} K\left(\mu_{1}, \lambda_{1}\right)$

where

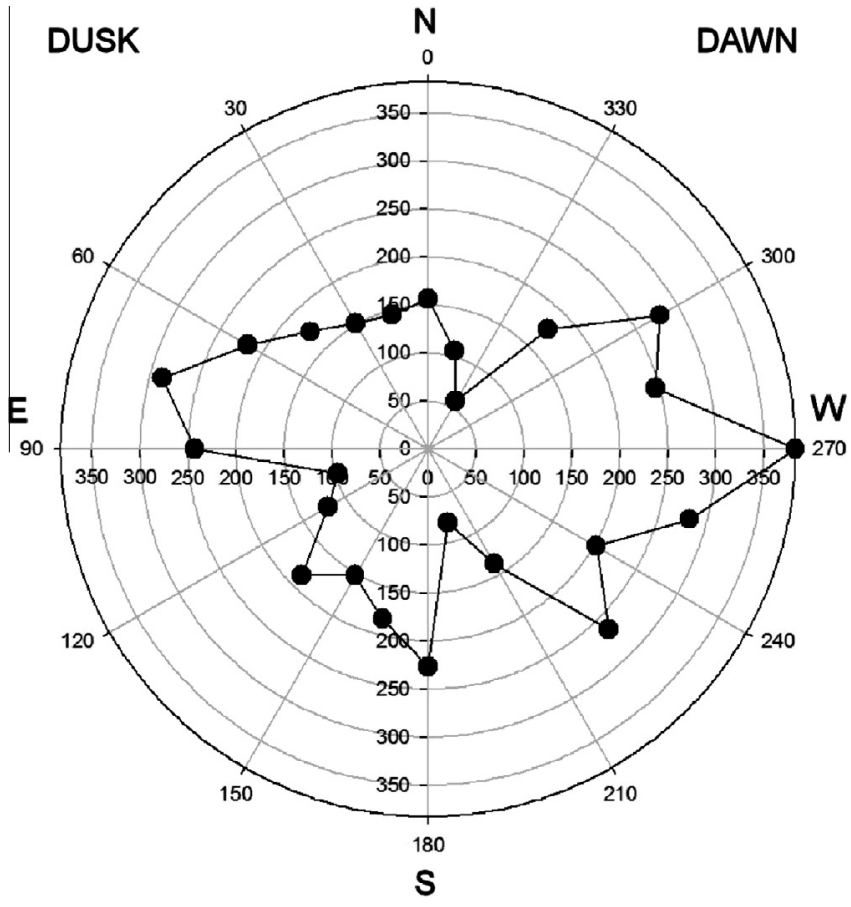

Fig. 14. This polar plot shows corrected scale heights in units of kilometers as a function of position angle around the disk of Mercury. The average scale height on the dawn hemisphere is about $100 \mathrm{~km}$ larger than on the dusk hemisphere. Values in this plot were taken from Table 2.

$K\left(\mu_{1}, \lambda_{1}\right)=\int_{0}^{\lambda_{1}} \frac{\lambda_{1}^{2} \varsigma(\lambda) e^{-\left(\lambda_{1}-\lambda\right)} d \lambda}{\lambda^{2} \varsigma\left(\lambda_{1}\right)\left[1-\left(1-\mu_{1}^{2}\right) \lambda^{2} / \lambda_{1}^{2}\right]^{1 / 2}}$

and $H_{1}=\frac{r_{1}}{\lambda_{1}}=\frac{k T_{c}}{\operatorname{Mg}\left(r_{1}\right)}, \lambda$ is the gravitational potential energy in units of $\mathrm{kT}, \mathrm{g}$ is the gravitational acceleration at $r_{1}, M$ is the mass of the atom, and $\mu=\cos (\theta)$.

A line of sight through the corona from the outside traverses an integrated density $2 N\left(\mu=0, \lambda_{1}\right)$. 


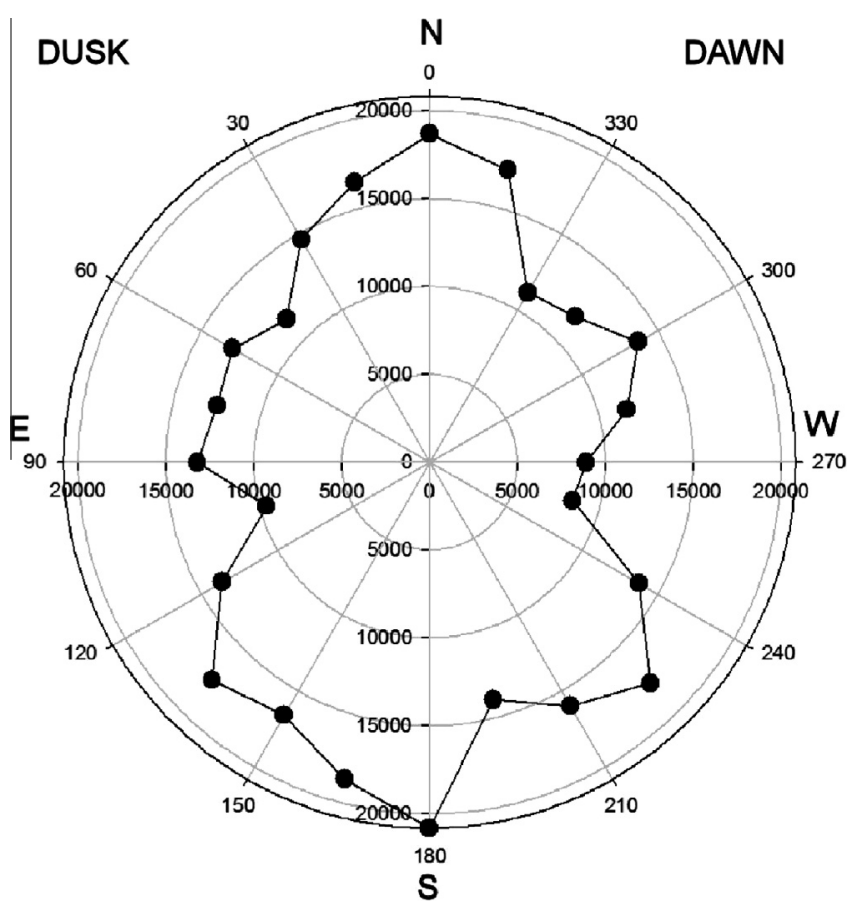

Fig. 15. Assuming that we have derived the true altitude distribution of equivalent width, the total sodium absorption can be calculated by integrating equivalent width as a function of altitude. This figure shows a polar plot of the total equivalent width as a function of position angle around the planet. Values in this plot were taken from Table 2 .

This formulation corrects for the barometric law (which is integrated over all momentum coordinates) because at substantial distances above the exobase the entire range of momenta are not allowed. However, it does not account for loss mechanisms other than collisions, such as radiation pressure and ionization. Thus we find that the actual distribution falls off more steeply with distance than this formulation, especially above $600 \mathrm{~km}$. The measured height distributions all curve slightly downward relative to the Chamberlain formulation but this is expected.

We computed tangent, or line-of-sight, column densities as a function of altitude from the surface equivalent widths and scale heights listed in Table 2, using the conversion factor between equivalent width and column density noted above. We then used the Chamberlain formulation to compute tangent column densities as a function of altitude for a series of models specifying only the surface number density and temperature. The code determines the best-fit solution by performing a least-squares analysis over the range of models. We then plotted the data and models in order to see how well the best-fit solution actually matched the data. In some cases we had to run more models because the initial guesses for number density and temperature did not encompass a wide enough range.

An example of the fitting procedure is shown in Fig. 16 for the sector at $180^{\circ}$, the south polar region.

Combinations of surface density and temperature are used to calculate the altitude profile, and then varied until a best-fit with the observed profile is reached. This procedure does not lend itself to straightforward calculation of errors. However, a reasonable error estimate would be $\pm 100^{\circ}$ for temperature and $\pm 200 \mathrm{~km}$ for altitude.

The fitting procedure described above yielded the surface density and the temperature for each sector around the planet. We calculated the scale height and zenith column density from these two factors assuming the barometric formula to hold. Table 3 lists the results.

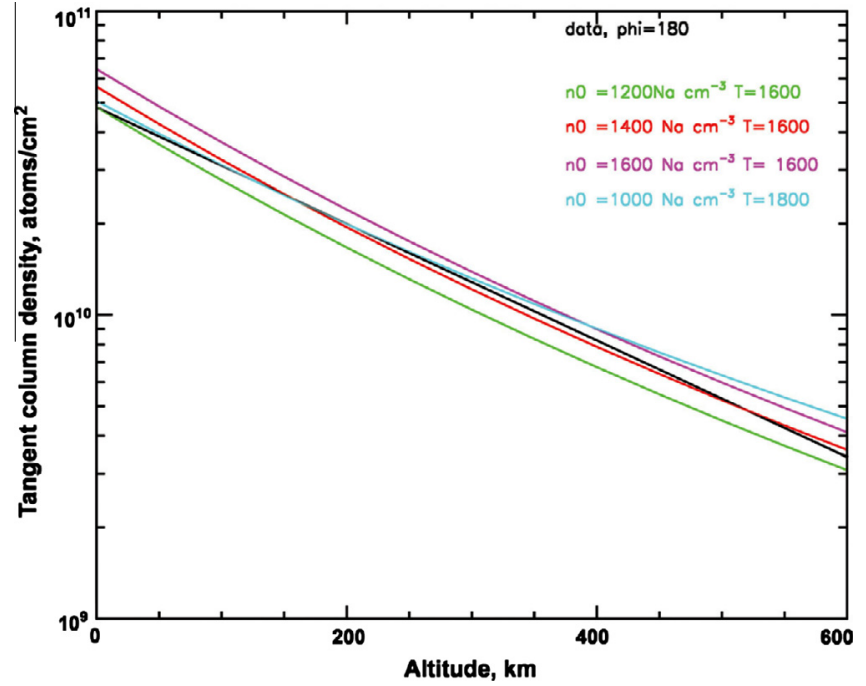

Fig. 16. Trial-and-error fitting of surface density and temperature to the observation, denoted by the black line in the figure. The best-fit was a density of 1400 atoms $/ \mathrm{cm}^{3}$ and a temperature of $1600 \mathrm{~K}$, which is the red line in the figure. (For interpretation of the references to color in this figure legend, the reader is referred to the web version of this article.)

The average surface concentration of sodium atoms was about 2700 atoms $/ \mathrm{cm}^{3}$, but high northern $\left(330-15^{\circ}\right)$ and southern latitudes $\left(195-210^{\circ}\right)$ were much denser. The average vertical column density was $2.7 \times 10^{10}$ atoms $/ \mathrm{cm}^{2}$, and the average scale height was $133 \mathrm{~km}$. The average temperature was about $1600 \mathrm{~K}$ on the dawn side, and about $1300 \mathrm{~K}$ on the dusk side. Killen et al. (1999) did a careful analysis of high-resolution sodium $\mathrm{D}_{2}$ emission line shapes, and reported sodium temperatures of $750 \mathrm{~K}$ at the poles and $1100 \mathrm{~K}$ at the equator, which are lower than our transit values. However, these observations are for an area of surface, not the terminator. Because of radiation pressure the scale height would increase toward the terminators observed in the transit data.

Fig. 17 shows a polar plot of the zenith column density around the circumference of the planet.

The column density reaches minima at the equatorial dusk and dawn terminators, with the dusk terminator column density larger than the dawn terminator column density. Maxima are reached in high-latitude polar regions.

A polar plot of temperatures against position angle is shown in Fig. 18.

Temperatures reach maxima across the equatorial regions, and minima in the polar regions. The average temperature on the dawn hemisphere is about $1600 \mathrm{~K}$, while the dusk hemisphere temperatures average smaller values, in the range of $1300 \mathrm{~K}$.

\subsubsection{Comparison with previous results}

The 2003 transit was observed by Schleicher et al. (2004), using instrumentation similar to that employed for this research. The general appearance of our equivalent width map is similar to that reported by Schleicher et al. (2004), who reported values ranging from $1 \mathrm{~mA}$ to $15 \mathrm{~mA}$ for the 2003 transit, similar to the values reported here, which ranged from 2 to $18 \mathrm{~mA}$.

Schleicher et al. (2004) found the sodium density near the equator at the dusk terminator to be at the noise level of their measurement, much smaller than the sodium density at the dawn terminator. However, we found a different result, in that the dusk terminator sodium density was slightly larger than the dawn terminator sodium. The average difference over a $60^{\circ}$ arc centered on the equator was about $10 \%$. It would appear that the direction 
Table 3

Surface densities, temperatures, scale heights and zenith column densities for the Mercury exosphere derived from corrected line-of-sight column densities.

\begin{tabular}{|c|c|c|c|c|}
\hline Position angle $\left({ }^{\circ}\right)$ & Surface density number $\left(\mathrm{cm}^{3}\right)$ & Temperature (K) & Scale height $(\mathrm{km})$ & Zenith column density number $\left(\mathrm{cm}^{2}\right)$ \\
\hline ON & 3200 & 1200 & 114.1 & $3.7 E+10$ \\
\hline 15 & 5100 & 1000 & 95.1 & $4.9 \mathrm{E}+10$ \\
\hline 30 & 2500 & 1200 & 114.1 & $2.9 \mathrm{E}+10$ \\
\hline 45 & 1600 & 1300 & 123.6 & $2.0 \mathrm{E}+10$ \\
\hline 60 & 900 & 1600 & 152.2 & $1.4 \mathrm{E}+10$ \\
\hline 75 & 600 & 1800 & 171.2 & $1.0 \mathrm{E}+10$ \\
\hline $90 \mathrm{E}$ & 600 & 1800 & 171.2 & $1.0 \mathrm{E}+10$ \\
\hline 105 & 5800 & 900 & 85.6 & $5.0 \mathrm{E}+10$ \\
\hline 120 & 5000 & 1100 & 104.6 & $5.2 \mathrm{E}+10$ \\
\hline 135 & 1800 & 1400 & 133.1 & $2.4 \mathrm{E}+10$ \\
\hline 150 & 2800 & 1200 & 114.1 & $3.2 \mathrm{E}+10$ \\
\hline 165 & 1800 & 1400 & 133.1 & $2.4 \mathrm{E}+10$ \\
\hline $180 \mathrm{~S}$ & 1400 & 1600 & 152.2 & $2.1 \mathrm{E}+10$ \\
\hline 195 & 4700 & 800 & 76.1 & $3.6 \mathrm{E}+10$ \\
\hline 210 & 3500 & 1100 & 104.6 & $3.7 \mathrm{E}+10$ \\
\hline 225 & 700 & 1900 & 180.7 & $1.3 \mathrm{E}+10$ \\
\hline 240 & 1100 & 1500 & 142.6 & $1.6 \mathrm{E}+10$ \\
\hline 255 & 300 & 2000 & 190.1 & $5.7 \mathrm{E}+09$ \\
\hline $270 W$ & 100 & 3000 & 285.3 & $2.9 \mathrm{E}+09$ \\
\hline 285 & 500 & 1800 & 171.2 & $8.6 \mathrm{E}+09$ \\
\hline 300 & 600 & 1800 & 171.2 & $1.0 \mathrm{E}+10$ \\
\hline 315 & 1100 & 1400 & 133.1 & $1.5 \mathrm{E}+10$ \\
\hline 330 & 10000 & 500 & 47.5 & $4.8 \mathrm{E}+10$ \\
\hline 345 & 9500 & 900 & 85.6 & $8.1 \mathrm{E}+10$ \\
\hline
\end{tabular}

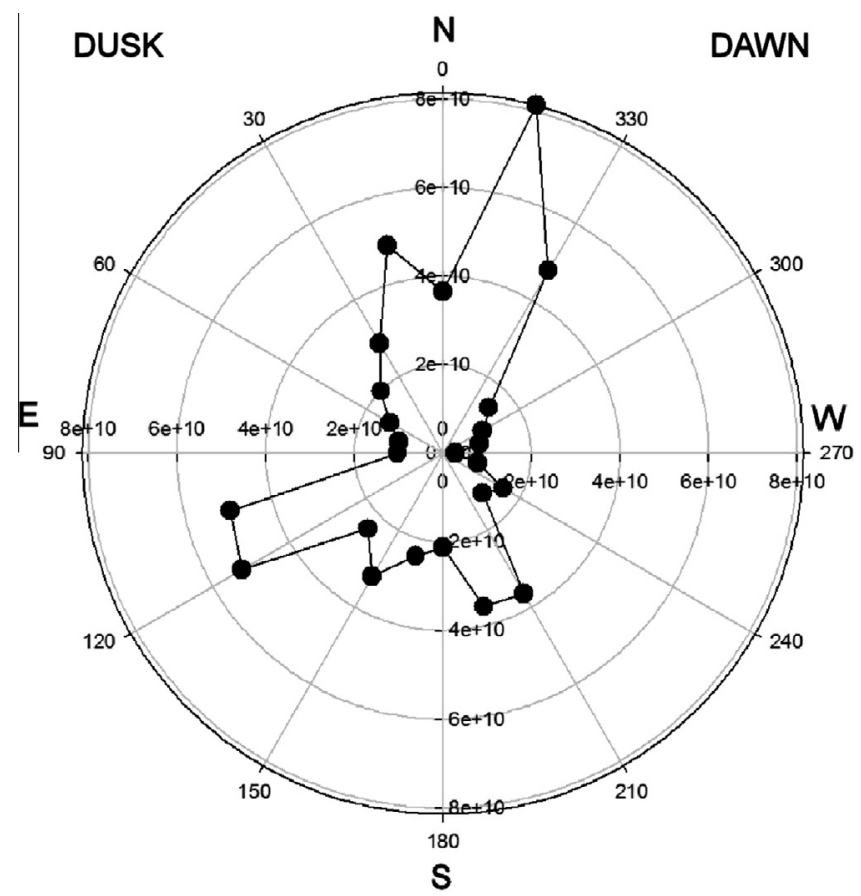

Fig. 17. The zenith column density in units of $10^{10}$ atoms $/ \mathrm{cm}^{2}$ is plotted against position angle around Mercury. The average column density on the dawn side was about $0.9 \times 10^{10}$ atoms $/ \mathrm{cm}^{2}$ and about $1.1 \times 10^{10}$ atoms $/ \mathrm{cm}^{2}$ on the dusk side. Values in this plot were taken from Table 3.

of the dawn to dusk terminator ratio observed by Schleicher et al. (2004) is consistent with the observations of Hunten and Sprague (2002), who found that sodium densities towards the dusk terminator were less than towards the dawn terminator, at least for the range of true anomaly angles that they explored.

However, Potter et al. (2006) measured terminator brightening over a near-complete range of true anomaly angles, comparing sodium densities for the terminator half of the illuminated planet with densities for the limb half. They found that the terminator

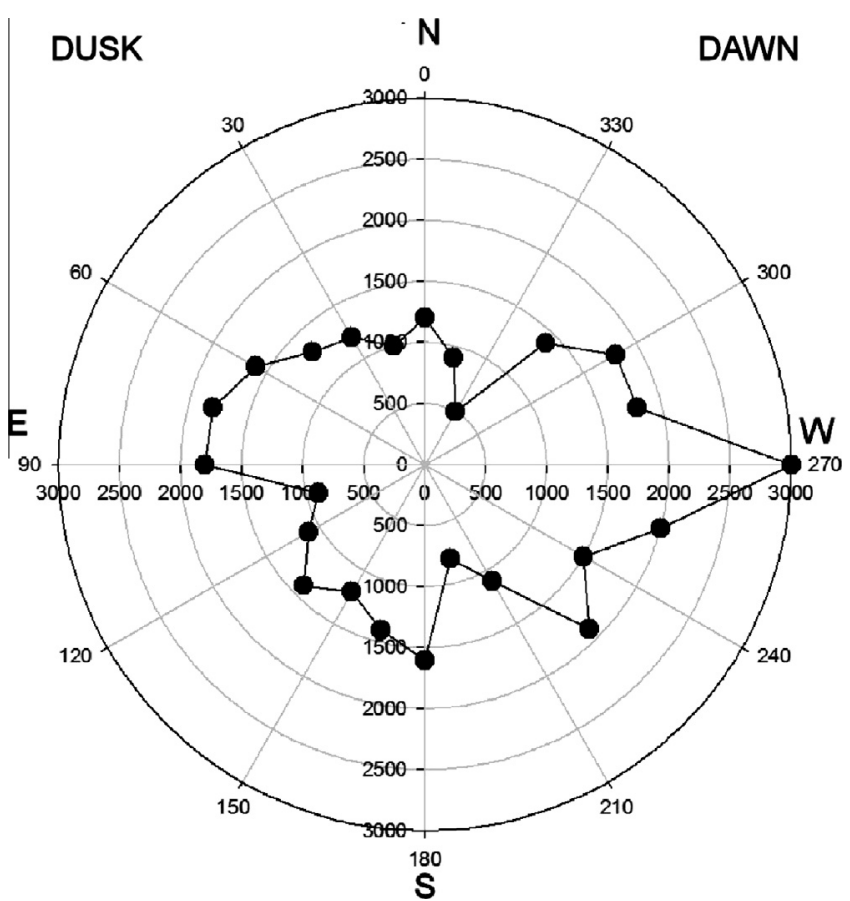

Fig. 18. Temperatures in units of degrees Kelvin are plotted against position angles around the planet. The average temperature on the dawn side was about $1600 \mathrm{~K}$, and on the dusk side was about $1300 \mathrm{~K}$. Values in this plot were taken from Table 3.

to limb ratio was large only in regions with high radiation pressure, primarily on the "out" leg of the orbit.

Large values of radiation pressure readily move sodium across the Mercury surface towards the terminators (Potter et al., 2009). There will be a tendency to accumulate sodium towards the terminator. At the dawn terminator, sodium will condense and then evaporate, returning to the exosphere as the planet rotates, resulting in terminator brightening. The opposite is true at the dusk terminator, where sodium will condense beyond the terminator, but 
will not return to the exosphere as the planet rotates, resulting in terminator dimming.

Radiation pressure at the time of the 2003 transit was about $14 \%$ of surface gravity, well into the region where radiation pressure is shown in Fig. 19 to have little or no effect on limb brightening. Consequently, the result of Schleicher et al. (2004) is not consistent with the terminator brightening observations of Potter et al. (2006).

The 2006 transit occurred at a time when Mercury was moving in towards the Sun. Then, solar radiation pressure is less effective than on the "out" leg of the orbit, since the pressure decreases as the atom velocity increases, rather than increasing, as happens on the "out" leg. The data in Fig. 19 are sparse on the "in" leg of the orbit, but terminator brightening is weak or non-existent over most of the "in" leg of the orbit. Radiation pressure at the time of the 2006 transit was $27 \%$ of surface gravity at about $30^{\circ}$ from aphelion on the "in" leg of the orbit. There is not much evidence for terminator brightening in that region. It appears that the nearequality of the dusk and dawn terminators observed for the 2006 transit is consistent with the observations of Potter et al. (2006).

There must be some other explanation for the terminator brightening observed by Schleicher et al. (2004). If there is a reservoir of condensed sodium on the night side, rotation of the dark cold surface into sunlight would produce a local enhancement even in the absence of any accumulation from radiation pressure. Consequently, we do expect some enhancement of sodium at the dawn terminator from this source as the Sun rises. If this effect covers only a narrow region near the terminator, the procedure used to calculate the ratios shown in Fig. 19 might not reveal its existence at a level exceeding the measurement error. The model of Mercury's exosphere for the 2003 transit developed by Mura et al. (2009) showed a small, narrow enhancement above the dawn terminator, which probably would not have been detected by the observations of Potter et al. (2006). (Effects of radiation pressure on sodium distribution were not included in the model.)

It is reasonable to expect that the size of the sodium cloud produced by solely by evaporation with little influence of radiation pressure would depend on the rate of exposure of fresh cold surface to dawn sunlight. There is a major difference between the 2003 and 2006 transits in the rate of advance of surface material at the terminator line into sunlight. This is a consequence of the

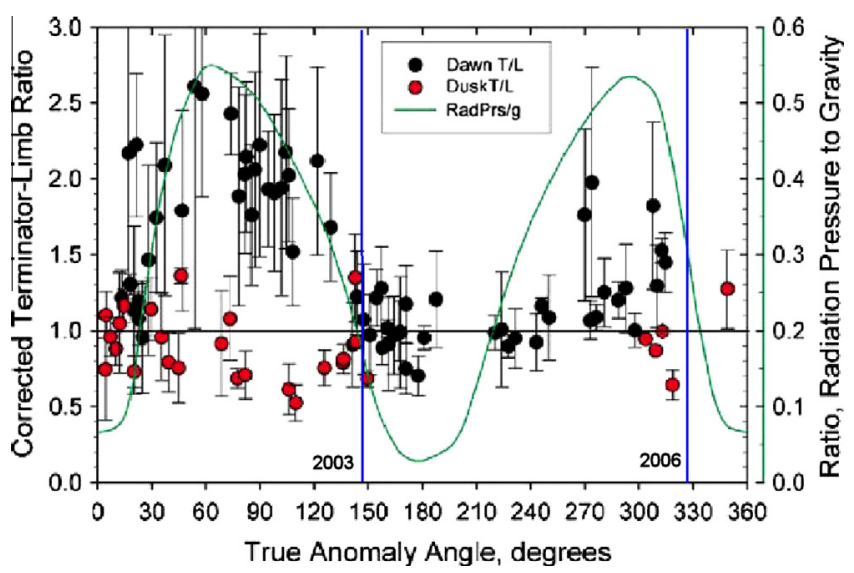

Fig. 19. Published data for terminator-to-limb brightness ratios as a function of true anomaly angle (from Potter et al. (2006). The dawn terminator-to-limb ratio is a maximum and the dusk terminator-to-limb ratio is a minimum near the point of maximum radiation pressure. In regions of low radiation pressure, the terminatorto-limb ratios are all near unity within scatter of the data. The true anomaly points for the 2003 transit $\left(149^{\circ}\right)$ and the 2006 transit $\left(328^{\circ}\right)$ are marked by vertical blue lines. (For interpretation of the references to color in this figure legend, the reader is referred to the web version of this article.) highly elliptic orbit of Mercury, as the result of which, there is a period near perihelion where the Sun moves retrograde across the Mercury sky. At the time of the 2003 transit, cold surface rotated into sunlight at the rate of $3.27^{\circ}(138.3 \mathrm{~km})$ per Earth day, near the maximum value of $3.4^{\circ}$ per Earth day at aphelion. Any dawn enhancement due to evaporation of night-side sodium would be near to a maximum. Sodium at the dusk terminator would be lost by condensation.

For the transit in 2006, the true anomaly angle midway through the transit was $329.3^{\circ}$, and the rate of advance of surface into sunshine at the dawn terminator was just $0.13^{\circ}(5.5 \mathrm{~km})$ per Earth day. Any dawn enhancement due to evaporation of night-side sodium would be near to a minimum and any dusk terminator loss would also be near a minimum. This is consistent with the model of the sodium exosphere developed by Leblanc and Johnson (2003), who showed that the dawn enhancement tended to vanish as perihelion was approached.

The difference between terminator effects observed by Schleicher et al. (2004) and those of this research can be reconciled if the rotation effect dominates conditions at the terminators.

Schleicher et al. (2004) reported the appearance of a streamerlike feature extending a thousand kilometers above the north polar region, and a similar but smaller feature above the south polar region. We found similar features above both the south and north polar regions, with more of them above the south polar regions, as shown in Figs. 4 and 5. An example of the altitude distribution for one of these features is shown in Figs. 7 and 8. We suggest that the sodium seen in these features may be the small population of high-energy $(\sim 3 \mathrm{eV})$ sodium that can be swept off the planet by solar radiation pressure to generate the Mercury tail, as identified by Potter and Killen (2008). This sodium may be produced from the surface by solar particle or electron sputtering. The solar radiation pressure at the time of the transit was not sufficient to generate an appreciable tail, so this population of sodium would remain in the exosphere.

We also consider that these high-altitude features may just be coincidental alignments of background "solar noise" at suitable heights above the limb. These high-altitude features could also be consistent with the magnitude $(1.6 \mathrm{~m} \AA)$ and spatial structure of the noise we derived from our data in regions far away from the planet. While Schleicher et al. (2004) do not provide similar noise level estimates, their streamer-like features are most likely also consistent with this same background noise. Atmospheric smearing of the stronger absorption at the poles could help bring borderline patches of solar structure in those regions above the defined noise threshold, preferentially enhancing the appearance of streamer like structures in the polar regions. Future transit experiments should seek to further reduce the effects of this background solar noise.

Schleicher et al. (2004) derived scale heights, surface number densities and zenith column densities at the northern and southern maxima. These are compared with results from this research in Table 4.

In general, there is fair agreement between the two sets of results. Effects of planetary rotation on the sodium exosphere at the terminator that we suggest caused the differences at the equator should be at a minimum near the poles.

Yoshikawa et al. (2008) observed the 2006 transit, and provided column density values of $4.1 \times 10^{10}$ for the "westside" and $6.1 \times 10^{10}$ atoms $/ \mathrm{cm}^{2}$ for the "eastside" without explanation as to how these values were derived from their observations of equivalent width. No uncertainty bands were provided for these data. They identified the "westside" as the dusk terminator and the "eastside" as the dawn terminator. This is incorrect if their directions were for Mercury as seen in the sky. It would be correct if their directions were for an individual standing on the planet. They 
Table 4

Comparison of results from this research with those from Schleicher et al. (2004).

\begin{tabular}{|c|c|c|c|c|}
\hline Observer & Position & Scale height (km) & Surface density $\left(10^{3}\right.$ atoms $\left./ \mathrm{cm}^{3}\right)$ & Column density $\left(10^{10}\right.$ atoms $\left./ \mathrm{cm}^{2}\right)$ \\
\hline Schleicher et al. (2004) & $\mathrm{N}$ & $135 \pm 30$ & 2.6 & 3.4 \\
\hline This research & $\mathrm{N}$ & $114 \pm 10$ & $3.2 \pm 0.3$ & $3.7 \pm 0.3$ \\
\hline Schleicher et al. (2004) & $\mathrm{S}$ & $130 \pm 30$ & 2.9 & 3.0 \\
\hline This research & $\mathrm{S}$ & $152 \pm 10$ & $1.4 \pm 0.3$ & $2.1 \pm 0.3$ \\
\hline
\end{tabular}

did not specify which situation was valid for their data. In any case, their results show that the relative dawn and dusk sodium absorptions are within about a 30\% range, which is consistent with our observations, but different from that seen for the 2003 transit, where the dusk terminator signal was vanishingly small.

Since no conversion factor for equivalent width to column density was given, and no error bounds were specified, we cannot determine if our measured equivalent width values are within or without the margin of error for their values. However, we can use our conversion factor to find values of equivalent width that would correspond to the reported column densities, which we assume are tangent column densities. The result was that their measured equivalent widths would be 78 and $116 \mathrm{~mA}$. These values are about an order of magnitude larger than those reported here for the observational data and similarly are much larger than those reported by Schleicher et al. (2004).

\subsection{Line widths for sodium absorption}

Fig. 20 shows a map of the Gaussian line widths around the circumference of Mercury.

The most common line width was $24 \mathrm{~mA}$, maximum line width was $36 \mathrm{~mA}$, and minimum line width was $20 \mathrm{~mA}$. The instrumental profile was $24 \mathrm{~mA}$, so that the true line widths are smaller, ranging from about $16 \mathrm{~mA}$ to $31 \mathrm{~mA}$.

Below about $200 \mathrm{~km}$, the corrected line widths are in the range 17 to $21 \mathrm{~mA}$, and are fairly uniform around the planet. These widths represent relatively low temperatures, less than or equal to about $1000 \mathrm{~K}$, consistent with PSD or ESD (Madey et al., 1998). These temperatures are close to the $750-1100 \mathrm{~K}$ values determined by Killen et al. (1999) from analysis of emission lines. At higher

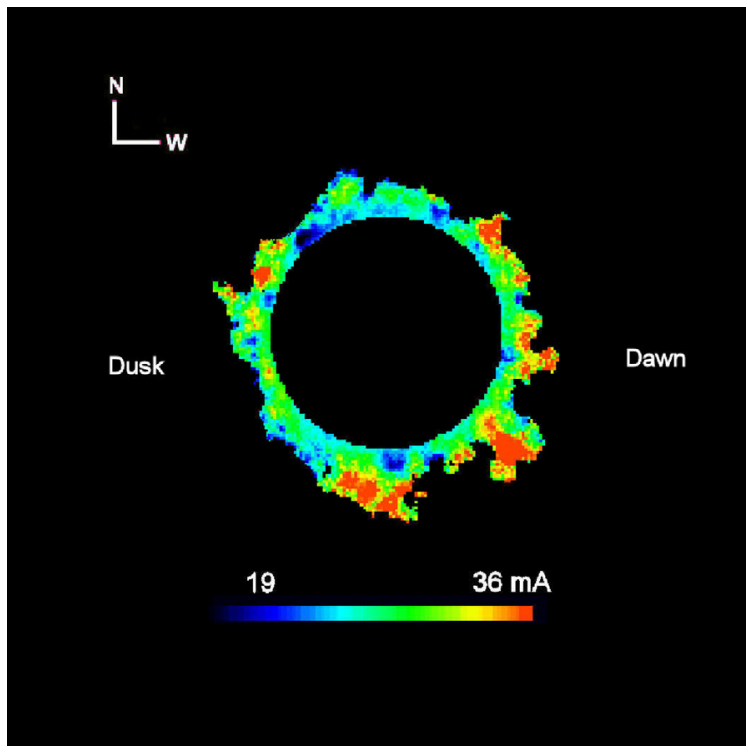

Fig. 20. Map of the Gaussian sigma width for the sodium absorption line around the circumference of the Mercury shadow. The average width was $24 \mathrm{~mA}$. The highest values of line width are above the south polar region and the dawn terminator altitudes, there are patches of broader lines. The regions which displayed high velocities above the south pole and dawn terminator show the widest lines, in the range 30-35 mA. These values represent temperatures in the range of $2000 \mathrm{~K}$. These regions are roughly coincident with the clouds illustrated in Fig. 7.

A close-up view of line widths in the sodium cloud above the surface in sector $225^{\circ}$ is shown in Fig. 21.

This is the same cloud for which equivalent widths were shown in Fig. 7. Line widths, and consequently temperatures, are at maximum values in part of the cloud, lending support to the suggestion that sodium in the cloud is a different population than sodium near the surface.

Schleicher et al. (2004) and Yoshikawa et al. (2008) observed line widths for the sodium absorption of $33 \mathrm{~mA}$ and $30 \mathrm{~mA}$ respectively. After correction for instrumental broadening, their average line widths were $31 \mathrm{~mA}$ and $25 \mathrm{~mA}$ respectively. These authors interpreted their line widths as resulting from intrinsic Doppler velocities, and obtained sodium temperatures of $3540 \mathrm{~K}$ (Schleicher) and $2237 \mathrm{~K}$ (Yoshikawa). Assuming a much lower temperature, as found by Potter and Morgan (1985), Schleicher et al. (2004) concluded that a non-thermal random velocity component of about $1 \mathrm{~km} / \mathrm{s}$ is mainly responsible for the observed line widths.

Yoshikawa et al. (2008) concluded that thermal desorption is the main source process for $\mathrm{Na}$ in the Hermean exosphere, in spite of having measured a temperature of $2237 \mathrm{~K}$, over 10 times the surface temperature at dawn and twice the temperature expected from a PSD source. Neither the width of the line, the scale height, nor the abundance is consistent with thermal desorption. The distribution of $\mathrm{Na}$ around the limb is inconsistent with a thermal source. Our derived temperatures, averaging $1600 \mathrm{~K}$ on the dawn side, and $1300 \mathrm{~K}$ on the dusk side, are consistent with photon-stimulated desorption

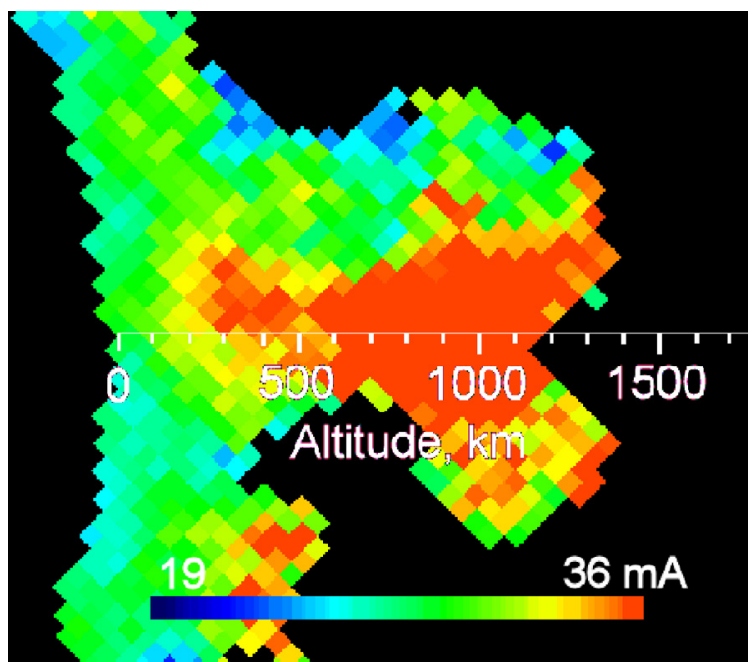

Fig. 21. This image displays the line widths in the sodium cloud displayed in the close-up view shown in Fig. 7. Line widths, and accordingly temperatures, are a maximum in the cloud, supporting the view that the sodium in this cloud is a different population from sodium near the surface. 
and electron-stimulated desorption as the primary source of sodium in the Mercury exosphere.

\subsection{Earthward velocities of sodium atoms}

Sodium atoms move across the surface by series of ballistic hops, and the atoms are accelerated antisunward by solar radiation pressure when aloft. For the transit observation, Mercury is observed exactly in front of the Sun, so that the velocity vector of sodium accelerated by solar radiation is directed at the Earth. Fig. 22 shows a map of Earthward velocities of the sodium atoms in the exosphere. Over most of the planet, velocities average $0.8 \mathrm{~km} / \mathrm{s}$.

From a study of sodium emission as a function of true anomaly angle, Potter et al. (2007) estimated that sodium atoms are accelerated for an average time of $1700 \mathrm{~s}$ (this was based on the difference in sodium emission intensity between in- and out-legs of the orbit). At the time of the 2006 transit, the solar radiation pressure for a sodium atom was $119 \mathrm{~cm} / \mathrm{s}^{2}$, which would produce an Earthward velocity of $1.5 \mathrm{~km} / \mathrm{s}$ for an acceleration period of $1700 \mathrm{~s}$, taking into account the decreases in radiation pressure that take place as the antisunward velocity increases. This is about twice the observed average velocity of $0.8 \mathrm{~km} / \mathrm{s}$. The effective acceleration time to produce a velocity of $0.8 \mathrm{~km} / \mathrm{s}$ would be about $600 \mathrm{~s}$, and this may represent the average time between hops near the terminator, assuming a strong interaction of sodium atoms with the surface.

While most velocities were near $0.8 \mathrm{~km} / \mathrm{s}$, there were two high velocity regions, averaging $1.3-1.5 \mathrm{~km} / \mathrm{s}$. One is found high over the South Pole, the other high over the dawn terminator region. These may represent sodium that has been projected high enough that surface interactions have not affected the acceleration by solar pressure. Sodium may be generated with high energies and projected up into the exosphere at high latitudes by particle or electron sputtering processes (Vervack et al., 2010; Potter and Killen, 2008), and this may explain the high velocity sodium seen over the south polar region, although a similar effect is not seen over the north pole. The equivalent width map shown in Fig. 4 shows several high altitude regions in south polar regions, and two in north polar regions. Low-latitude high velocities on the dawn side may have originated from high-energy sodium produced from

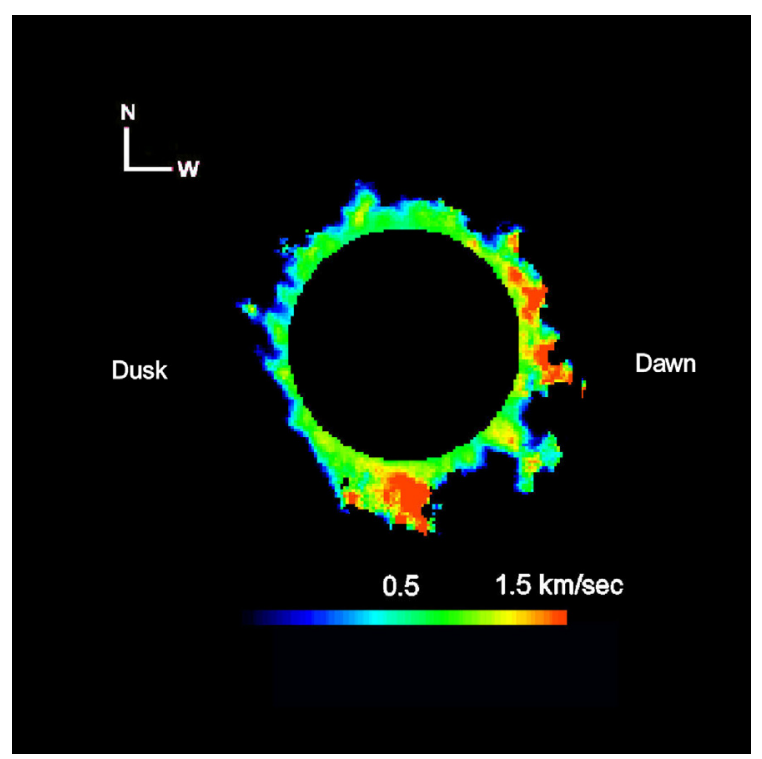

Fig. 22. Map of the Earthward velocities of sodium around the circumference of the Mercury shadow. The average velocity in this figure is $0.8 \mathrm{~km} / \mathrm{s}$ towards Earth Standard deviation of velocities is $0.25 \mathrm{~km} / \mathrm{s}$. The highest velocities are found over the south pole and the dawn terminator. night side equatorial region sputtering (Benna et al., 2010). The highest sodium temperature found from the Chamberlain analysis was $3000 \mathrm{~K}$ over the dawn side.

It should be noted that the radiation pressure at the time of this observation was insufficient to generate an appreciable sodium tail, whereby substantial numbers of sodium atoms escape the planet (Potter and Killen, 2008; Schmidt et al., 2012). Potter and Killen (2008) found that for true anomaly angles greater than $180^{\circ}$ (the "in" leg), the tail could no longer be detected at radiation pressures of $121 \mathrm{~cm} / \mathrm{s}^{2}$ or less. Radiation pressure at the time of the transit was $119 \mathrm{~cm} / \mathrm{s}^{2}$ and the true anomaly was $329.3^{\circ}$, so that sodium in the tail should have been present only in very small amounts. Had an appreciable tail been present, velocities seen down through the tail would have been much larger, due to the long times that escaped sodium atoms are accelerated by solar radiation pressure.

\section{Concluding remarks}

We measured sodium absorption around the disk of Mercury during its transit across the Sun on November 6, 2006. We derived the surface density, temperature, zenith column density and scale height of exospheric sodium in the altitude range from the surface up to about $600 \mathrm{~km}$. We have estimated the noise level in our measurement and corrected for the inherent smearing by the Earth's atmosphere. The column densities over the dawn equatorial terminator were found to be smaller than over the dusk equatorial terminator, opposite from the difference reported for the transit of 2003 by Schleicher et al. (2004). Assuming these results to be valid, the difference between the two observations could have resulted from the slow rate at which the dawn terminator advanced into sunlight during the 2006 transit relative to the more rapid rate during the 2003 transit. Comprehensive modeling of the sodium exosphere at the dusk and dawn terminators over the complete range of true anomaly angles might provide additional insight.

At altitudes above $600 \mathrm{~km}$, there was small population of energetic sodium in the north and south polar regions, identified as a population of sodium with sufficient energy to be swept off the planet into the tail at sufficiently high radiation pressures, evidence of a different source process at the polar regions.

We derived higher temperatures on the dawn-side for the sodium exosphere. Although the temperatures are quite different for Na and Ca, MESSENGER observations of Ca in the Mercury exosphere indicate that high-temperature $\mathrm{Ca}$ is produced almost exclusively over the dawn hemisphere (Burger et al., 2011, 2012).

The circum-Mercury pattern of derived temperatures matched closely the circum-Mercury pattern of line widths, with high temperatures corresponding to wide lines, and low temperatures corresponding to relatively narrow lines. Earthward velocities of the sodium atoms averaged about $0.8 \mathrm{~km} / \mathrm{s}$, about half the predicted value.

The error budgets listed for the results shown in the tables and figures were all derived from the noise level in the solar background on which the Mercury sodium absorption was superimposed. There are other potential sources of error that we cannot account for at the present time. For example, Fig. 6 shows a semi logarithmic plot of equivalent width against altitude. A straight line has been fitted to the line, and this line fit is the basis for all of the analysis that follows. However, the data depart slightly from the straight line starting around $300 \mathrm{~km}$, rising above it, and dropping below it at higher altitudes. While these deviations are small, they would be considerably magnified by a true correction for seeing blur, suggesting that the altitude distribution is more complex than assumed for the data analysis.

Another uncertainty has to do with the fact that the observations are of the terminator region. This is a region of change, where radiation pressure causes sodium atoms to flow from sunlit regions 
to dark regions where they may condense, and where condensed sodium atoms may be released into the exosphere as the surface warms in dawn sunlight. As a consequence, values for atmospheric parameters derived from the terminator data should not be considered representative of the whole planet without taking into account the effect of terminator processes. For example, the large difference between dawn and dusk terminators observed by Schleicher et al. (2004) is surely not representative of the whole planet, but rather, in part, the effect of terminator processes.

Observation of sodium absorption in the exosphere of Mercury during a solar transit provides unique information about the planetary distribution of sodium and its sources. The next two transits, in 2016 and 2019, will be visible in Europe, and it is hoped that similar observations will be possible, perhaps extended to additional species such as $\mathrm{Ca}$ or $\mathrm{K}$ in the Mercury exosphere.

\section{Acknowledgments}

The National Solar Observatory is operated by the Association of Universities for Research in Astronomy under a cooperative agreement with the National Science Foundation. The IBIS instrument used for the transit observations at Sunspot, New Mexico, was built by the Arcetri Astrophysical Observatory and the Department of Astronomy and Space Science of the Florence University, in collaboration with the Department of Physics of the Roma "Tor Vergata" University. We thank the Community Coordinated Modeling Center (CCMC) at NASA Goddard Space Flight Center for running the ENLIL model to estimate the solar wind and interplanetary magnetic field parameters at the time of our observations. RMK acknowledges support from NASA under the MESSENGER Participating Scientist Program and NASA Planetary Astronomy grants. We also thank Jay Pasachoff, Bryce Babcock of Williams College, as well as Fabio Cavallini and Antonio Vecchio of Arcetri Observatory, for their assistance in planning the observations. We are very grateful to Doug Gilliam, Mike Bradford, and Joe Elrod and the NSO staff for all their outsized efforts in preparing for and carrying out the observations at Sunspot, New Mexico. We thank Claude Plymate, Eric Galayda and Matthew Knight for supporting the transit observations that were made with the McMath-Pierce Solar Telescope at Kitt Peak, Arizona, using the Main Spectrograph. We also thank the reviewers, who provided insightful comments that improved the quality of this paper.

\section{References}

Anderson, B.J. et al., 2011. The global magnetic field of Mercury from MESSENGER orbital observations. Science 333, 1859. http://dx.doi.org/10.1126/ science.1211001, \# 6051.

Baumgardner, J., Wilson, J.K., Mendillo, M., 2008. Imaging the sources and full extent of the sodium tail of the planet Mercury. Geophys. Res. Lett. 35. http:/ dx.doi.org/10.1029/2007GL032337, \# 3, CiteID L03201.

Benna, M.The MESSENGER AMDG Team, 2010. Modeling of the magnetosphere of Mercury at the time of the first MESSENGER flyby. Icarus 209, 3-10. http:// dx.doi.org/10.1016/j.icarus.2009.11.036.

Burger, M.H. et al., 2011. Calcium in Mercury's Exosphere: Modeling MESSENGER Data. Am. Geophys. Union (Fall Meet.). Abstract \#P41A-1596.

Burger, M.H. et al., 2012. Modeling MESSENGER observations of calcium in Mercury's exosphere. J. Geophys. Res. 117. http://dx.doi.org/10.1029/ 2012JE004158, CiteID E00L11 (in press).

Cavallini, F., 2006. IBIS: A new post-focus instrument for solar imaging spectroscopy. Solar Phys. 236, 415-439.

Cavallini, F., Reardon, K., 2006. IBIS instrumental characteristics and first results. Mem. Soc. Astron. Ital., Suppl. 9, 55-58.

Chamberlain, J.W., Hunten, D.M., 1987. Theory of Planetary Atmospheres: An Introduction to their Physics and Chemistry,... International Geophysics Series, second ed., vol. 36. Academic Press, Orlando, pp. 331-351 (Chapter 7 Stability of planetary atmospheres, Section 7.1 Quasi-collisionless exospheres).

Chance, K., Kurucz, R.L., 2010. An improved high-resolution solar reference spectrum for Earth's atmosphere measurements in the ultraviolet, visible, and near infrared. J. Quant. Spectrosc. Radiat. Trans. 111, 1289-1295.

Evans, L.G., Peplowski, P.N., Killen, R.M., Potter, A.E., Sprague, A.L., 2013. Variable sodium on the surface of Mercury: Implications for surface chemistry and the exosphere. Lunar Planet. Sci. 44. Houston, TX. LPL Contribution No. 1719.
Ho, G.C. et al., 2012. Spatial distribution and spectral characteristics of energetic electrons in Mercury's magnetosphere. J. Geophys. Res. 117. http://dx.doi.org/ 10.1029/2012JA017983. A00M04.

Hunten, D.M., Sprague, A.L., 2002. Diurnal variation of Na and K at Mercury. Meteort. Planet. Sci. 37, 1191-1195.

Killen, R.M., Potter, A., Fitzsimmons, A., Morgan, T.H., 1999. Sodium $\mathrm{D}_{2}$ line profiles: Clues to the temperature structure of Mercury's exosphere. Planet. Space Sci. 47, 1449-1458.

Killen, R.M., Shemansky, D.E., Mouawad, N., 2009. Expected emission from Mercury's exospheric species, and their UV-visible signatures. Astrophys. J., Suppl. 181, 351-359, \# 2.

Leblanc, F., Johnson, R.E., 2003. Mercury's sodium exosphere. Icarus 164, 261-268. Leblanc, F., Johnson, R.E., 2010. Mercury exosphere I. Global circulation model of its sodium component. Icarus 209, 280-300.

Leblanc, F. et al., 2009a. High latitude peaks in Mercury's sodium exosphere: Spectral signature using THEMIS. Geophys. Res. Lett. 35. http://dx.doi.org/ 10.1029/2008GL035322, \# 18.

Leblanc, F. et al., 2009b. Short-term variations of Mercury's Na exosphere observed with very high spectral resolution. Geophys. Res. Lett. 36. http://dx.doi.org/ 10.1029/2009GL038089, \# 7, CiteID L07201.

Madey, T.E., Yakshinskiy, B.V., Ageev, V.N., Johnson, R.E., 1998. Desorption of alkali atoms and ions from oxide surfaces-Relevance to origins of $\mathrm{Na}$ and $\mathrm{K}$ in atmospheres of Mercury and the Moon. J. Geophys. Res. 103, 5873-5887.

Mangano, V., Leblanc, F., Barbieri, C., Massetti, S., Milillo, A., Cremonese, G., Grava, G., 2009. Detection of a southern peak in Mercury's sodium exosphere with the TNG in 2005. Icarus 201, 424-431. http://dx.doi.org/10.1016/ j.icarus.2009.01.016, \# 2.

Mura, A., Wurz, P., Lichtenegger, H.I.M., Schleicher, H., Lammer, H., Delcourt, D., Milillo, A., Orsini, S., Massetti, S., Khodachenko, M.L., 2009. The sodium exosphere of Mercury: Comparison between observations during Mercury's transit and model results. Icarus 200, 1-11.

Potter, A.E., Killen, R.M., 2008. Observations of the sodium tail of Mercury. Icarus $194,1-12$.

Potter, A., Morgan, T., 1985. Discovery of sodium in the atmosphere of Mercury. Science 229, 651-653.

Potter, A.E., Talent, D., Kurokawa, H., Kawakami, S., Morgan, T.H., 1994. Attempt to observe the sodium exosphere of Mercury during the 1993 solar transit. Lunar Planet Sci. 25, 1099

Potter, A.E., Killen, R.M., Morgan, T.H., 2000. Variation of lunar sodium during passage of the Moon through the Earth's magnetotail. J. Geophys. Res. 105, 15073-15084.

Potter, A.E., Killen, R.M., Sarantos, M., 2006. Spatial distribution of sodium on Mercury. Icarus $181,1-12$

Potter, A.E., Killen, R.M., Morgan, T.H., 2007. Solar radiation acceleration effects on Mercury sodium emission. Icarus 186, 571-580.

Potter, A.E., Morgan, T.H., Killen, R.M., 2009. Sodium winds on Mercury. Icarus 204, 355-367.

Press, W.H., Teukolsky, S.A., Vetterling, W.T., Flannery, B.R., 1992. Numerical Recipes in Fortran, second ed., pp 530-538, Press Syndicate of the University of Cambridge, 40 West 20th Street, New York, NY 1011-4211 USA.

Reardon, K., Cavallini, F., 2008. Characterization of Fabry-Perot interferometers and multi-etalon transmission profiles. The IBIS instrumental profile. Astron. Astrophys. 481, 897-912.

Righini, A., Cavallini, F., Reardon, K.P., 2010. Imaging performance of multi-etalon bidimensional spectrometers. Astron. Astrophys. 515. http://dx.doi.org/ 10.1051/0004-6361/200913302, ID A85.

Rimmele, T.R., 2004. Recent advances in solar adaptive optics. In: Calia, Domenico B., Ellerbroek, Brent L., Ragazzoni, Roberto (Eds.), Advancements in Adaptive Optics. Proceedings of the SPIE, vol. 5490, pp. 34-46. SPIE Press, 1000 20th St., Bellingham, WA, 98225, USA

Sarantos, M. Killen, R.M., Sharma, A.S., Slavin, J.A. 2010. Sources of sodium in the lunar exosphere: Modeling using ground-based observations of sodium emission and spacecraft data of the plasma. Icarus 205, 364-374. http:// dx.doi.org/10.1016/j.icarus.2009.07.039, \# 2.

Schleicher, H., Wiedemann, G., Wöhl, H., Berkefeld, T., Soltau, D., 2004. Detection of neutral sodium above Mercury during the transit on 2003 May 7. Astron. Astrophys. 425, 1119-1124.

Schmidt, C.A., Wilson, J.K., Baumgardner, J., Mendillo, M., 2010. Orbital effects on Mercury's escaping sodium exosphere. Icarus 207, 9-16. http://dx.doi.org/ 10.1016/j.icarus.2009.10.017, \# 1.

Schmidt, C.A., Baumgardner, J., Mendillo, M., Wilson, J.K., 2012. Escape rates and variability constraints for high-energy sodium sources at Mercury. J. Geophys. Res. 117, A3. http://dx.doi.org/10.1029/2011JA017217, CiteID A03301.

Vecchio, A. et al., 2005a. Periodic behavior and stochastic fluctuations of solar activity: Proper orthogonal decomposition analysis. Solar Phys. 229, 359-372, \# 2.

Vecchio, A. et al., 2005b. Proper orthogonal decomposition of solar photospheric motions. Phys. Rev. Lett. 95, \# 6, ID 061102-1-061102-4.

Vervack Jr., Ronald J. et al., 2010. Mercury's complex exosphere: Results from MESSENGER's third Flyby. Science 329, 672-675.

Winslow, R.M. et al., 2012. Mercury's magnetopause and bow shock from MESSENGER observations. J. Geophys. Res.. http://dx.doi.org/10.1002/ jgra.50237.

Yoshikawa, I. et al., 2008. Observation of Mercury's sodium exosphere during the transit on November 9, 2006. Planet. Space Sci. 56, 1676-1680. 